\title{
The structure of shock waves as a test of Brenner's modifications to the Navier-Stokes equations
}

\author{
CHRISTOPHER J. GREENSHIELDS \\ AND JASON M. REESE† \\ Department of Mechanical Engineering, University of Strathclyde, \\ Glasgow G1 1XJ, United Kingdom
}

(Received 10 August 2006 and in revised form 5 October 2006)

Brenner $(2005 a, 2005 b)$ has recently proposed modifications to the Navier-Stokes equations that are based on theoretical arguments but supported only by experiments having a fairly limited range. These modifications relate to a diffusion of fluid volume that would be significant for flows with high density gradients. So the viscous structure of shock waves in gases should provide an excellent test case for this new model. In this paper we detail the shock structure problem and propose exponents for the gas viscosity-temperature relation based on empirical viscosity data that is independent of shock experiments. We then simulate monatomic gas shocks in the range Mach 1.0-12.0 using the Navier-Stokes equations, both with and without Brenner's modifications. Initial simulations showed Brenner's modifications display unphysical behaviour when the coefficient of volume diffusion exceeds the kinematic viscosity. Our subsequent analyses attribute this behaviour to both an instability to temporal disturbances and a spurious phase velocity-frequency relationship. On equating the volume diffusivity to the kinematic viscosity, however, we find the results with Brenner's modifications are significantly better than those of the standard Navier-Stokes equations, and broadly similar to those from the family of extended hydrodynamic models that includes the Burnett equations. Brenner's modifications add only two terms to the Navier-Stokes equations, and the numerical implementation is much simpler than conventional extended hydrodynamic models, particularly in respect of boundary conditions. We recommend further investigation and testing on a number of different benchmark non-equilibrium flow cases.

\section{Introduction}

The generally-accepted parameter which indicates the extent to which a local region of flowing gas is in thermodynamic equilibrium is the Knudsen number:

$$
K n=\frac{\lambda}{L} \propto \frac{M a}{R e},
$$

where $\lambda$ is the mean free path of the gas molecules, $L$ is a characteristic length of the flow system, the Mach number of the flow $M a=|\boldsymbol{u}| / c$ with $\boldsymbol{u}$ the flow velocity and $c$ the speed of sound, and the Reynolds number $R e=\rho|\boldsymbol{u}| L / \mu$ with $\rho$ the mass density and $\mu$ the dynamic viscosity. For high Re flows over solid bodies, the characteristic length scale is the boundary layer thickness, and the denominator on the right hand side of 
(1.1) is $\sqrt{R e}$ (see, e.g., Schaaf \& Chambré 1961). As Kn increases, the departure of the gas from local thermodynamic equilibrium increases, and the notion of the gas as a continuum-equilibrium fluid becomes less valid. The range of use of the continuumequilibrium assumption is therefore clearly limited, with the applicability of the classical Navier-Stokes-Fourier equations (with standard no-slip boundary conditions) confined to cases where $K n \lesssim 0.01$, typically. Extended, or modified, hydrodynamics attempts to extend the range of applicability of the continuum-equilibrium fluid model into the so-called 'intermediate- $K n$ ' (or 'transition-continuum') regime where $0.01 \lesssim K n \lesssim 1$.

Brenner (2005a) recently proposed modifications to the Navier-Stokes-Fourier equations for flows with appreciable density gradients. His theoretical developments and experimental validations are centred on slow moving flows where variations in density are primarily caused by variations in temperature rather than pressure. Of particular interest to Brenner is the motion of particles due to thermal gradients, called 'thermophoresis', which provides good, yet fairly limited, supporting evidence for his work. This range of evidence should be broadened, particularly since his work challenges the fundamentals of conventional fluid dynamics and so demands rigorous validation. It is therefore of particular interest to see whether his modifications to the classical Navier-Stokes-Fourier equations improve their predictive capabilities for intermediate- $K n$ flows.

In this paper, we investigate the application of Brenner's modified Navier-Stokes equations to the shock structure problem. This case has been used in the past for testing several proposed extended hydrodynamic models, such as the Burnett equations, by Muckenfuss (1962), Bird (1970), Barcelo (1971), Steinhilper (1972), Sturtevant \& Steinhilper (1986), Alsmeyer (1976), Narasimha \& Das (1986), Lumpkin \& Chapman (1991), Zhong et al. (1991), Reese (1993), Reese et al. (1995), Levermore \& Morokoff (1998), Cercignani et al. (1999), Myong (2001), Macrossan \& Lilley (2003), Xu \& Tang (2004), Torrilhon \& Struchtrup (2004) and Struchtrup (2005). Brenner's modified equations can be considered an extended hydrodynamic model, and their relationship to established models is discussed towards the end of this paper. In order to be concise and avoid ambiguity, for the remainder of this paper we shall use the expression "Navier-Stokes" to refer to the classical Navier-Stokes-Fourier equation set, and adopt the term "Brenner-NavierStokes" to refer to Brenner's modified version of these.

\section{The shock structure problem}

The shock structure problem concerns the spatial variation in fluid flow properties across a stationary, planar, one-dimensional shock in a monatomic gas. We define the flow as moving at a speed $u$ in the positive $x$-direction, with the shock located at $x=0$; the upstream conditions at $x=-\infty$ are super/hypersonic and denoted by a subscript ' 1 ', downstream conditions at $x=+\infty$ are denoted by a subscript ' 2 '. While shocks are often modelled as discontinuities, their physical properties in fact vary continuously from their upstream to their downstream levels over a characteristic distance of a few mean free paths because the relaxation times for heat and momentum transport are finite. The flow in this shock layer is far from being in local thermodynamic equilibrium, typically $K n=0.2 \sim 0.3$, i.e. very much within the intermediate- $K n$ regime.

Since the Navier-Stokes equations perform poorly at these $K n$, the shock structure problem is particularly apposite for testing extended hydrodynamic models. The problem possesses certain features that make it attractive for numerical investigation, particularly if hydrodynamic models with high-order derivatives are used:

(a) there are no solid boundaries to consider;

(b) the upstream and downstream boundary conditions are clearly defined through 
the Rankine-Hugoniot relations, with all gradients of flow quantities tending to zero far upstream and downstream of the shock;

(c) the problem is one-dimensional and steady.

A monatomic gas possesses no modes of vibration or rotation, cannot dissociate, and only ionizes at the highest temperatures, so its thermodynamic behaviour is generally much simpler than that of polyatomic gases. It is for this reason that monatomic gases have generally been preferred as the test gas in shock structure experiments and analysis. Resulting data have historically been presented in normalised form and the lack of access to the raw data presents an opportunity for us to specify the test problem in a nondimensionalised form that reduces the pre- and post-processing effort $\dagger$ :

- It is convenient to set the upstream temperature $T_{1}=1$ because, for a given $\mu_{1}$, the coefficient of proportionality $A$ is then independent of the exponent of $s$ in the power law relation of (3.3). For simplicity we then choose $A=1$ and, hence, $\mu_{1}=1$.

- It is useful to set $c_{1}=1$ so that the upstream flow speed $u_{1}$ relates directly to the upstream Mach number $M a_{1}$. The ratio of specific heats, $\gamma$, is $5 / 3$ for monatomic gases, so the gas constant $R=c^{2} /(\gamma T)=3 / 5$ in our adopted nondimensionalised set of units.

- The final parameters can be set so that a unit length corresponds to the upstream Maxwellian mean free path:

$$
\lambda_{M 1}=\frac{16}{5 \sqrt{2 \pi \gamma}} \frac{\mu_{1} c_{1}}{p_{1}} .
$$

where $p_{1}$ is the upstream pressure. For this test case $16 / 5 \sqrt{2 \pi \gamma}=0.99 \approx 1$, so $\lambda_{M 1} \approx$ $\mu_{1} c_{1} / p_{1}$. By setting $p_{1}=1$, the unit length then corresponds almost exactly to $\lambda_{M 1}$.

All normal gradients of $p$ and $T$ are specified as zero at the solution domain boundary far downstream. The shock is maintained stationary and fixed within the domain by application of the Rankine-Hugoniot velocity relation at the downstream boundary, which for our case is

$$
\frac{u_{2}}{u_{1}}=\frac{1}{4}\left(1+3 M a_{1}^{-2}\right) .
$$

The case is initialised with a step change in fields from upstream to downstream at $x=0$. To minimise the time required to reach a steady-state solution, the downstream pressure and temperature are initialised using Rankine-Hugoniot relations for pressure and temperature, which for our case are:

$$
\frac{p_{2}}{p_{1}}=\frac{1}{4}\left(5 M a_{1}^{2}-1\right) \quad \text { and } \quad \frac{T_{2}}{T_{1}}=\frac{p_{2}}{p_{1}} \frac{u_{2}}{u_{1}} .
$$

The initial and boundary conditions for the actual solution variables, described in section 4 below, are simply derived from those for $p, T$ and $\boldsymbol{u}$, e.g. initial and boundary conditions for $\rho$ are calculated from $p$ and $T$ using the perfect gas law, $p=\rho R T$. When we include the second derivative of $\rho$ through application of (4.7), below, we specify additionally that the normal gradient of the gradient of $\rho$ is zero.

\section{The viscosity-temperature relation}

One of the main uncertainties in the physical modelling of the shock structure problem is the relation between $\mu$ and $T$. This is unfortunate because the $\mu(T)$ relation has an appreciable effect on the profile of a simulated shock - in the extreme case of assuming a constant $\mu$, results of simulations are very poor. It could even be argued that the

$\dagger$ In what follows, the use of a power law viscosity model, described in section 3.1 , is anticipated. 
Navier-Stokes equations could be made to work for the shock structure problem simply by adjusting the $\mu(T)$ relation until experimental shock profiles are reproduced. The value of the shock structure problem as a good test for hydrodynamic models therefore relies on establishing a good $\mu(T)$ relation from reliable experimental sources, preferably independent of shock data. We therefore review sources to establish $\mu(T)$ relations for a range of monatomic gases, from which argon is chosen for our test problem.

\subsection{The power law relation}

The viscosity of a perfect rarefied gas is defined through

$$
\mu=\tau_{1} p \propto \tau_{1} n T=\frac{5}{4} \tau_{0} n T,
$$

where $n$ is the gas molecular number density, $\tau_{1}$ is the collision interval for momentum transport, and $\tau_{0}$ is the collision interval for hard-sphere molecules. That the viscosity given in (3.1) is, in fact, purely dependent on temperature and not mass density, arises from the nature of the intermolecular force law which determines how molecules interact in collision with each other. For reasons of simplicity, this force is often modelled, for a given species of molecule at a particular temperature, as varying with distance from the molecular centre as an inverse power law with coefficient $\nu$. In a collision, molecules approach each other with a relative speed $g$ and slow to a stop a distance $d$ from each other when their kinetic energy is transformed to potential energy in the force field, i.e. $g^{2} \propto$ $d^{-\nu+1}$. With translational temperature a function of the square of the molecular velocity, it is then clear that the effective molecular diameter, $d \propto T^{-1 /(\nu-1)}$. The collisional relaxation time $\tau_{0}$ is then the mean free path $\left(\propto n^{-1} d^{-2}\right)$, divided by the mean molecular velocity $\left(\propto T^{1 / 2}\right)$ so that

$$
n \tau_{0} \propto T^{-1 / 2} d^{-2}=T^{s-1}, \quad \text { where } \quad s=\frac{1}{2}+\frac{2}{\nu-1} .
$$

Equations (3.1) and (3.2) yield the well-known relation

$$
\mu \propto T^{s} \quad \text { or } \quad \mu=A T^{s},
$$

where $A$ is a constant of proportionality. There are two theoretical limiting cases for the intermolecular force law: $\nu=\infty, s=1 / 2$ corresponds to hard-sphere molecules; $\nu=5$, $s=1$ corresponds to so-called Maxwellian molecules. Real molecules generally have a value of $\nu$ ranging from about 5 to around 15 .

\subsection{Experimental data for monatomic gases}

Increasing the value of the exponent $s$ in (3.3) in a shock structure calculation introduces more dissipation, particularly at the high-temperature, downstream side of the shock. This additional dissipation acts to smooth out the shock layer, increasing its thickness and, in particular, lengthening the downstream "tail" in the flow property profiles.

Therefore, in order to test any hydrodynamic model it is important to set the exponent $s$ independently of the shock structure problem under investigation. Maitland \& Smith (1972) critically assessed the viscosities of a number of gases, obtained from several different sources using a variety of techniques, such as the capillary flow method, oscillating disc and rotating cylinder methods, and observations of the retardation of an oil drop in free-fall through a gas. For the monatomic gases argon, helium and xenon they produced viscosity data which they estimated to be accurate to $1.5 \%$ in the temperature range $80-2000 \mathrm{~K}$. That upper limit of $2000 \mathrm{~K}$ is the downstream temperature of a shock of Mach 4.3 propagating into a room-temperature gas; hence these viscosity data are applicable to shocks of $M a \leq 4.3$. 


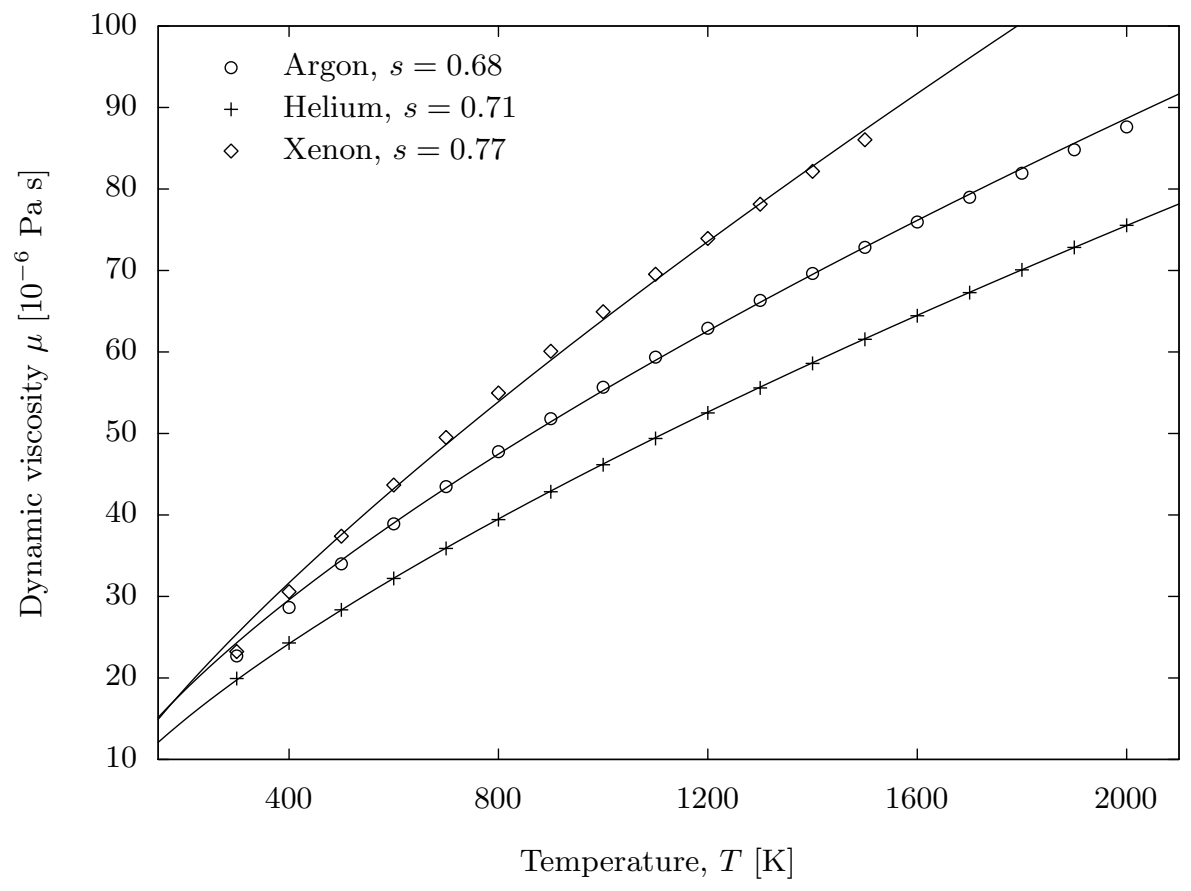

FIGURE 1. Experimental viscosity versus temperature data for intermediate temperatures for argon, helium and xenon, fitted to a power-law: $\mu \propto T^{s}$.

\begin{tabular}{lcc|cc|cc} 
& \multicolumn{2}{c}{ Argon } & \multicolumn{2}{c|}{ Helium } & \multicolumn{2}{c}{ Xenon } \\
\cline { 2 - 7 } Applicable range & $s$ & $\nu$ & $s$ & $\nu$ & $s$ & $\nu$ \\
up to Mach 4.3 & 0.68 & 12.0 & 0.71 & 10.7 & 0.77 & 8.5 \\
Mach 4.4-12.5 & 0.76 & 8.8 & 0.83 & 7.2 & 0.72 & 10.3
\end{tabular}

TABLE 1. Collated experimental values of $s$ and $\nu$ for argon, helium and xenon gases.

Amdur \& Mason (1958) estimated the intermolecular potential at higher temperatures from observations of the scattering of high-velocity molecular beams, and produced tables of the viscosity of gases at temperatures up to $15000 \mathrm{~K}$, corresponding to a shock of Mach 12.5. It is not known how accurate these data are; Amdur and Mason estimated that at the higher temperatures the error in viscosity could be as much as $10 \%$.

We have used these data to estimate the value of $s$ (and hence $\nu$ ) in (3.2) for different temperature ranges. We have fitted curves of the power law in (3.3) by minimising the error in viscosity for the two sets of experimental data. More details of this process can be found in Reese (1993), but the experimental data and best-fit curves for three common monatomic gases are shown in figures 1 and 2, and the corresponding exponents $s$ and $\nu$ are given in table 1.

The coefficient $\nu$ is itself a function of temperature because at higher temperatures molecules with more energy can penetrate each others' force-fields more effectively. Therefore, due to the differences in temperature range and experimental techniques reported in the Maitland \& Smith and Amdur \& Mason papers, we have considered two ranges of temperature or, equivalently, shock Mach number: up to Mach 4.3, and up to Mach 12.5. (It should be noted that the power-law fit is not very good for the high temperature 


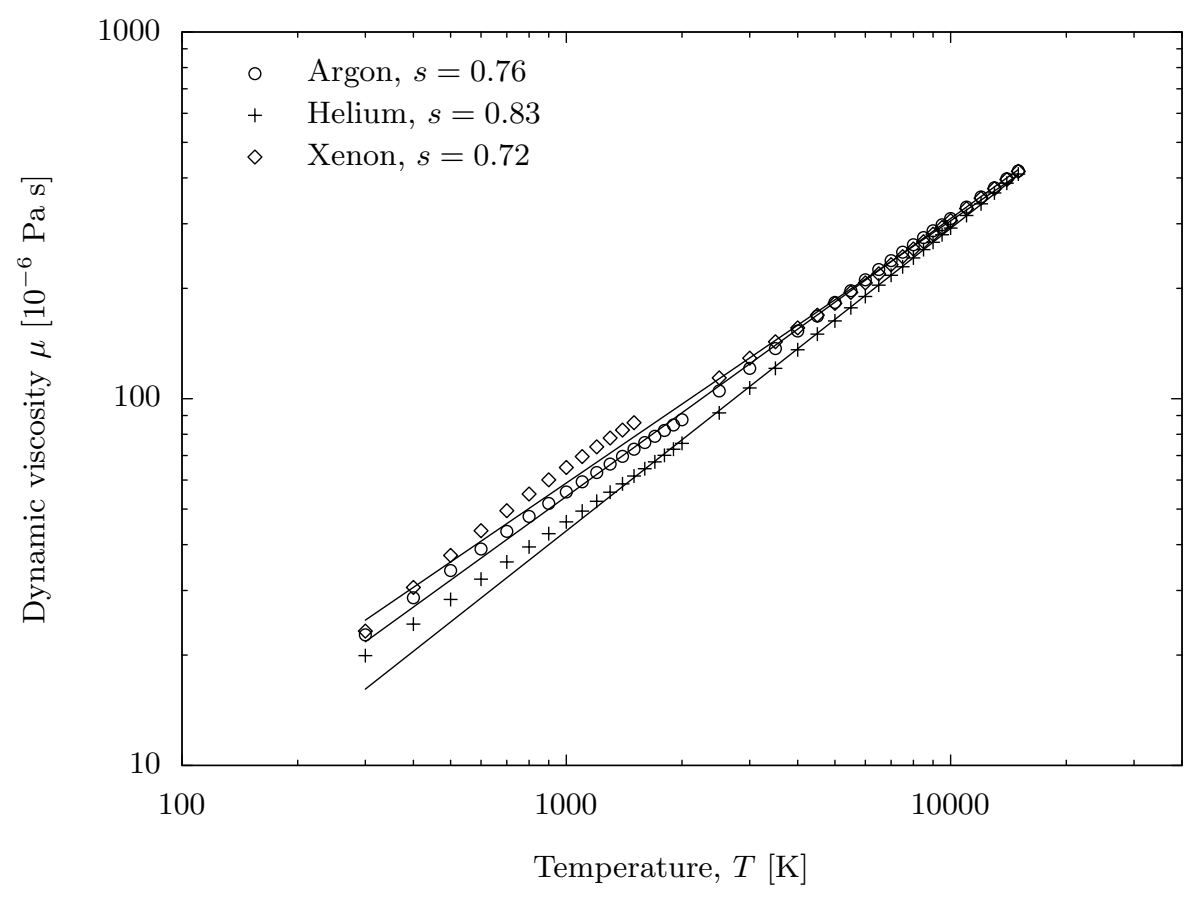

Figure 2. Experimental viscosity versus temperature data for high temperatures for argon, helium and xenon, fitted to a power-law: $\mu \propto T^{s}$.

data, which is itself of unknown accuracy, therefore the $\mu(T)$ obtained must be treated with caution.)

In the more limited temperature range of 3500-8500K, Aeschliman \& Cambel (1970) obtained values for argon viscosity to an accuracy of $12 \%$ which can be represented to within $1 \%$ error by a viscosity-temperature exponent of $s=0.74$. This value compares well with our value of $s=0.76$ in table 1 for temperatures in the range 2000-15000K.

Correlations between direct simulation Monte-Carlo (DSMC) simulations and experimental shock density profiles can also provide data for the exponent $s$, particularly at high temperatures (see Bird 1970; Barcelo 1971; Steinhilper 1972; Sturtevant \& Steinhilper 1986; Alsmeyer 1976; Lumpkin \& Chapman 1991; Macrossan \& Lilley 2003). While it is clearly preferable in our study of the shock structure problem to use data that are independent of the problem itself, it is worth noting that each of these published papers produces a value of $\nu$ that falls within a range $9 \leq \nu \leq 11$, corresponding to $0.70 \leq s \leq 0.75$. The value $s=0.72$ of Alsmeyer (1976) is often quoted: e.g. simulations by Macrossan \& Lilley (2003) using this value of $s$ agreed with experiment within an estimated uncertainty of $5 \%$ for temperatures above $2000 \mathrm{~K}$.

In our present study, three values of the exponent $s$ for argon are therefore used:

- $s=0.68$, which is our best-fit for shock Mach numbers up to 4.3;

- $s=0.76$, our best-fit up to Mach 12.5; and

- $s=0.72$, which is the mean of our best-fit values, as well as the commonly used value of Alsmeyer (1976) that falls in the middle of the range of values from DSMC correlations with shock density profiles. As this is the mean value, it is used as the 'control' in our study.

We focus on the power-law form of $\mu(T)$ in this paper in order to discern effects on shock structure due to different constitutive models for momentum and energy diffu- 
sion rather than due to the presumed relationship between gas properties. However, we recognise that there are other models available for $\mu(T)$, e.g. Sutherland's Law, that are generally equivalent to adding a weak attractive component to the intermolecular force - which is physically more realistic. In our simple power-law model, this attractive force would manifest itself as an exponent $s$ that decreases as temperature increases, which is generally reported (see for example Chapman \& Cowling 1970). It is interesting to note, however, that our present analysis of experimental data does not appear to bear this out: in the case of xenon in table $1, s$ decreases with increasing temperature, but with argon and helium the exponent increases with temperature.

\section{Brenner's modification to the Navier-Stokes equations}

Brenner presents his modification to the Navier-Stokes equations as a change in Newton's viscosity law. Before arriving at that discussion, we first express the standard governing equations in an Eulerian frame of reference as:

Conservation of mass

\section{Conservation of momentum}

$$
\frac{\partial \rho}{\partial t}+\nabla \cdot\left(\boldsymbol{u}_{m} \rho\right)=0
$$

$$
\frac{\partial \boldsymbol{m}}{\partial t}+\nabla \cdot\left(\boldsymbol{u}_{m} \boldsymbol{m}\right)+\nabla \cdot \boldsymbol{P}=0
$$

\section{Conservation of total energy}

$$
\frac{\partial E}{\partial t}+\nabla \cdot\left(\boldsymbol{u}_{m} E\right)+\nabla \cdot \boldsymbol{j}_{e}+\nabla \cdot\left(\boldsymbol{P} \cdot \boldsymbol{u}_{m}\right)=0
$$

where $\boldsymbol{u}_{m}$ is the mass velocity, the momentum density $\boldsymbol{m}=\rho \boldsymbol{u}_{m}$, the total energy density $E=\rho\left(e+\left|\boldsymbol{u}_{m}\right|^{2} / 2\right)$ with $e$ the specific internal energy, $\boldsymbol{j}_{e}$ is the diffusive flux density of internal energy, and $\boldsymbol{P}$ is the diffusive flux density of momentum — the familiar stress tensor - defined here as positive in compression: $\boldsymbol{P}=p \boldsymbol{I}+\boldsymbol{T}$, where $\boldsymbol{T}$ is the viscous stress tensor and $\boldsymbol{I}$ the unit tensor.

Based on this sign convention, the constitutive model for a Newtonian fluid relates $\boldsymbol{T}$ to the rate of deformation tensor, $\boldsymbol{D}$, by $\mu$ and the bulk viscosity $\kappa$ :

$$
\boldsymbol{T}=-2 \mu \stackrel{\circ}{\boldsymbol{D}}-\kappa \operatorname{tr}(\boldsymbol{D}) \boldsymbol{I}
$$

where $\boldsymbol{D} \equiv \overline{\nabla \boldsymbol{u}} \equiv \frac{1}{2}\left[\boldsymbol{\nabla} \boldsymbol{u}+(\boldsymbol{\nabla} \boldsymbol{u})^{\mathrm{T}}\right]$ for a velocity $\boldsymbol{u}$, i.e. the overbar indicates the symmetric part of a tensor. The deviatoric component of the deformation is $\stackrel{\circ}{\boldsymbol{D}} \equiv \boldsymbol{D}-$ $\frac{1}{3} \operatorname{tr}(\boldsymbol{D}) \boldsymbol{I}$.

In this constitutive model (4.4) it is generally considered that $\boldsymbol{u}$ is the mass velocity $\boldsymbol{u}_{m}$ that, in the mass continuity equation, relates to a convective flux of mass $\mathrm{d} \boldsymbol{S} \cdot \rho \boldsymbol{u}_{m}$ at an element of surface area $\mathrm{d} \boldsymbol{S}$, or that in the Boltzmann equation represents the statistical mean value velocity. However, this assumption has recently been questioned by Brenner $(2005 a, 2005 b)$ who postulated that the velocity appearing in Newton's viscosity law should instead be the volume velocity $\boldsymbol{u}_{v}$, so named since it relates to the flux of volume rather than mass.

The distinction between mass and volume flux is perhaps best explained by considering a single species fluid at a molecular level. The mass flux through $\mathrm{d} \boldsymbol{S}$ is the product of the molecular mass and the number of molecules passing through $\mathrm{d} \boldsymbol{S}$ in one second. The convective flux is defined such that there is no net mass flux due to random motions of molecules and, therefore, no diffusive mass flux. 
To understand volume flux we can consider attributing to each molecule locally a microscopic portion of the volume of fluid. This molecular volume is transported with the molecule but will change depending on the mass density of its surroundings, e.g. the microscopic volume of fluid shrinks as the molecule moves into a denser region. A convective flux of volume is associated with bulk motion of the molecular volumes, and is equivalent to the ratio of convective mass flux to mass density, i.e. $\mathrm{d} \boldsymbol{S} \cdot \boldsymbol{u}_{m}$. If the fluid density varies across $\mathrm{d} \boldsymbol{S}$, as a molecule passes through $\mathrm{d} \boldsymbol{S}$ there is a change in its associated volume, thus a net flux of volume. Random motions can therefore produce a net flux of volume, so that there exists a diffusive flux of volume in regions of non-zero density gradient. The volume flux $\mathrm{d} \boldsymbol{S} \cdot \boldsymbol{u}_{v}$ therefore represents the total flux of volume, comprising the convective flux d $\boldsymbol{S} \cdot \boldsymbol{u}_{m}$ and a diffusive flux $\mathrm{d} \boldsymbol{S} \cdot \boldsymbol{j}_{v}$, where $\boldsymbol{j}_{v}$ is the diffusive volume flux density, such that

$$
\boldsymbol{u}_{v}=\boldsymbol{u}_{m}+\boldsymbol{j}_{v}
$$

For a single component fluid undergoing heat transfer, Brenner (2005a) proposed a constitutive equation for $\boldsymbol{j}_{v}$ :

$$
\boldsymbol{j}_{v}=\alpha_{v} \frac{1}{\rho} \nabla \rho
$$

where $\alpha_{v}$ is termed the 'volume diffusivity'. Exactly how $\alpha_{v}$ should be quantified for a given fluid state is an open question. Brenner relates $\alpha_{v}$ directly to well-known diffusivity coefficients under some limited conditions; in particular, for single component fluids undergoing heat transfer, $\alpha_{v}$ is the same as the thermal diffusivity $\alpha=k / \rho c_{p}$, where $k$ is the thermal conductivity and $c_{p}$ is the specific heat capacity at constant pressure.

While the distinction between volume and mass velocities has been made, the question remains of why the velocity appearing in Newton's viscosity law should be the volume velocity rather than mass velocity. Brenner's justification is based on limited evidence (e.g. comparison of analytical solutions with thermophoresis experiments). A lack of theoretical physical argument could therefore lay the hypothesis open to some criticism. However, some support for it can be found within the phenomenological GENERIC theory presented by Öttinger (2005). First, Öttinger demonstrates that the GENERIC formulation arrives at the standard Navier-Stokes equations when the terms associated with mass density in the friction matrix are identically zero. Then, by including nonzero terms associated with mass density in the friction matrix, a revised set of governing equations is derived that includes two velocities, similar to $\boldsymbol{u}_{m}$ and $\boldsymbol{u}_{v}$ defined through (4.5) and (4.6). What emerges is that the standard governing equations have historically ignored mass diffusivity on the basis that the diffusive mass flux is zero, while forgetting that there are associated momentum and energy fluxes that may not be zero. GENERIC includes these momentum and energy fluxes, both of which are entropy producing, making the process of mass diffusion irreversible. The ability of mass diffusion to produce entropy is, according to Öttinger, something that is missing from the conventional Navier-Stokes equations.

Brenner's modification, essentially (4.5), can be incorporated into the system of governing fluid equations either by recasting the equations using $\boldsymbol{u}_{v}$ as the convective velocity instead of $\boldsymbol{u}_{m}$, or by using $\boldsymbol{u}_{v}$ in the constitutive equation for Newton's viscosity law. The former approach has been adopted elsewhere (see Öttinger 2005; Bardow \& Öttinger 2007) but here we choose the latter simply so that the Brenner modification appears more clearly as a new extended hydrodynamic model, rather than a radical change to the governing equations themselves. 
For a monatomic gas, $\kappa=0$. Combining (4.4), (4.5) and (4.6) yields a modified expression for the viscous stress:

$$
\boldsymbol{T}=-\mu\left[\boldsymbol{\nabla} \boldsymbol{u}_{m}+\left(\boldsymbol{\nabla} \boldsymbol{u}_{m}\right)^{\mathrm{T}}-\frac{2}{3} \boldsymbol{\nabla} \cdot \boldsymbol{u}_{m}\right]-2 \mu \overline{\boldsymbol{\nabla}\left\{\frac{\alpha}{\rho} \boldsymbol{\nabla} \rho\right\}}
$$

The Brenner approach requires the transport of energy to be similarly modified through consideration of the diffusion of internal energy. It is usually assumed that the diffusion of internal energy consists solely of heat diffusion, so that the diffusive internal energy flux density, $\boldsymbol{j}_{u}$, is considered synonymous with diffusive heat transfer, $\boldsymbol{q}=-k \boldsymbol{\nabla} T$, according to Fourier's law. However, the presence of a diffusive volume flux, of flux density $\boldsymbol{j}_{v}$, enables energy to be transported across a surface by diffusive work transfer of an amount $-p \boldsymbol{j}_{v}$. The diffusive internal energy flux density is therefore given by:

$$
\boldsymbol{j}_{e}=\boldsymbol{q}-p \boldsymbol{j}_{v},
$$

which, following our argument above, can be re-written in the form:

$$
j_{e}=-k \nabla T-\alpha_{v} \frac{p}{\rho} \nabla \rho .
$$

Equations (4.7) and (4.9) comprise Brenner's modifications to the classical NavierStokes-Fourier equations. We should note that this new fluid model has yet to receive either independent theoretical justification or experimental confirmation. As with any new hypothesis or model it is also subject to refinement and re-casting into different forms. However, we use it in this paper in the form presented in Brenner (2005b), without prejudice, to provide an indication of its current utility and limitations.

\section{Numerical solution of the governing equations}

Our numerical shock structure solver is developed using the open source Field Operation and Manipulation (OpenFOAM) software (see OpenCFD Ltd 2004). Written in $\mathrm{C}++$, OpenFOAM uses finite volume (FV) numerics to solve systems of partial differential equations ascribed on any 3-dimensional unstructured mesh of polygonal cells. All solvers developed within OpenFOAM are therefore 3-dimensional, but can be used for 1- or 2-dimensional problems by the application of particular conditions on boundaries lying in the plane of the direction(s) of no interest.

Fluid flow solvers in OpenFOAM are generally developed within an implicit, pressurevelocity, iterative solution framework. The solver we developed for this work first solves (4.1), (4.2) and (4.3) for $\rho, \boldsymbol{m}$ and $E$ respectively. The equations are treated in a segregated manner: for each equation, terms including the solution variable are, wherever possible, treated implicitly, with other terms treated explicitly. All equations include convection of transported variables that require a consistent, conservative set of fluxes of $\boldsymbol{u}_{m}$. After solving the sequence of segregated equations for $\rho, \boldsymbol{m}$ and $E$, an iterative PISO-style method (similar to Issa 1986) solves an equation for pressure $p$, derived from the perfect gas law, and (4.1) and (4.2), to produce conservative fluxes of $\boldsymbol{m}$ from which the fluxes of $\boldsymbol{u}_{m}$ are derived. Finally, $\boldsymbol{m}$ is also corrected from its new fluxes and $\rho$ is corrected from the new solution of $p$ according to the perfect gas law, before moving forward to the next time step and returning to the sequence of equations for $\rho, \boldsymbol{m}$ and E.

Our FV discretisation maintains a compact computational molecule for the orthogonal component of the Laplacian terms, which corresponds to the interpolation of Rhie \& 
Chow (1982) in the pressure equation. Both the transported fields in convection terms and the fluxes in $\boldsymbol{m}$ are interpolated using limiters from the MUSCL total variation diminishing (TVD) scheme (see van Leer 1979; Hirsch 1990) with identical limiters used in all convection terms (for $\rho, \boldsymbol{m}$ and $E$ ) to maintain numerical consistency. The temporal derivative is discretised using a two-time-level Euler implicit scheme.

We calculated shocks of Mach 1.2, 1.7, 2.2, 2.84, 3.4, 4, 5, 6, 7, 8, 9, 10 and 11 in order to provide a reasonable distribution of solution points for subsequent comparison with results from experiment. Mach 2.84 was specifically chosen to coincide with shock profile data communicated to us privately by Torecki \& Walenta (1993). Similarly, Mach 8 was chosen to coincide with the published shock profile data of Steinhilper (1972), and Mach 9 coincides with a published profile of Alsmeyer (1976).

A solution domain of $33 \lambda_{M 1}$ was used in all simulations — wide enough to contain the entire shock structure comfortably. Our initial results were obtained using the NavierStokes equations with the control viscosity exponent $s=0.72$. The results for $\rho$ and $T$ converged on a mesh of 800 cells to within $1 \%$ of the solution extrapolated to an infinitely small mesh size. The results we present in this paper were produced with a mesh of 2000 cells, corresponding to a mesh size of $\sim 0.017 \lambda_{M 1}$. Numerical solutions were executed until they converged to steady-state, at which point the residuals of all equations had fallen 5 orders of magnitude from their initial level.

\section{Volume diffusivity}

\subsection{Unphysical behaviour when $\alpha_{v} \equiv \alpha$}

We followed our initial Navier-Stokes simulations with preliminary simulations using the Brenner-Navier-Stokes equations. These simply used a volume diffusivity $\alpha_{v} \equiv \alpha$, which was Brenner's original suggestion (discussed in section 4 above). However, our simulations do not reach a converged solution with decreasing cell (or mesh) size: at the upstream edge of the temperature profile a small undershoot develops at a cell size of $0.06 \lambda_{M 1}$ that increases in magnitude with decreasing cell size. The problem is present in profiles of all solution variables but is best illustrated in a plot of Mach number, as shown in figure 3 for a Mach 8 shock. At best, the level of overshoot at the smallest cell sizes seems unphysical; worse is that the overshoot may tend to infinity as the mesh is further refined.

There is little doubt that the overshoot in Mach number is a consequence of Brenner's modification. In subsequent tests we were able to attribute the presence of the overshoot to the additional term in the momentum flux, but not to the additional term in the energy flux. We therefore postulate that the overshoot might be caused by an inappropriately large volume diffusivity, particularly since it exceeds the diffusivity associated with the remaining terms in the model, the kinematic viscosity $\nu=\mu / \rho$, by a factor $\alpha^{\star}=\alpha_{v} / \nu=$ $\operatorname{Pr}^{-1}=1.5$.

Our search for an alternative value of $\alpha_{v}$ began by relating the physical process of volume diffusion more closely to mass diffusion, rather than thermal diffusion. However, the process of diffusion of mass within a single component fluid, or self-diffusion, occurs at a similar rate to thermal diffusion. For a monatomic nonpolar gas, a self-diffusivity coefficient $D_{m} \approx 1.32 \nu$ can be derived from Chapman-Enskog theory using the LennardJones (6-12) potential (see Chapman \& Cowling 1970). The unphysical overshoot in Mach number remains for this value of self-diffusion coefficient. The overshoot becomes less pronounced as $\alpha_{v}$ is reduced but where the overshoot exists, it appears to be unphysical and may tend to infinity as the mesh is refined to an infinitesimally small size. Only when 


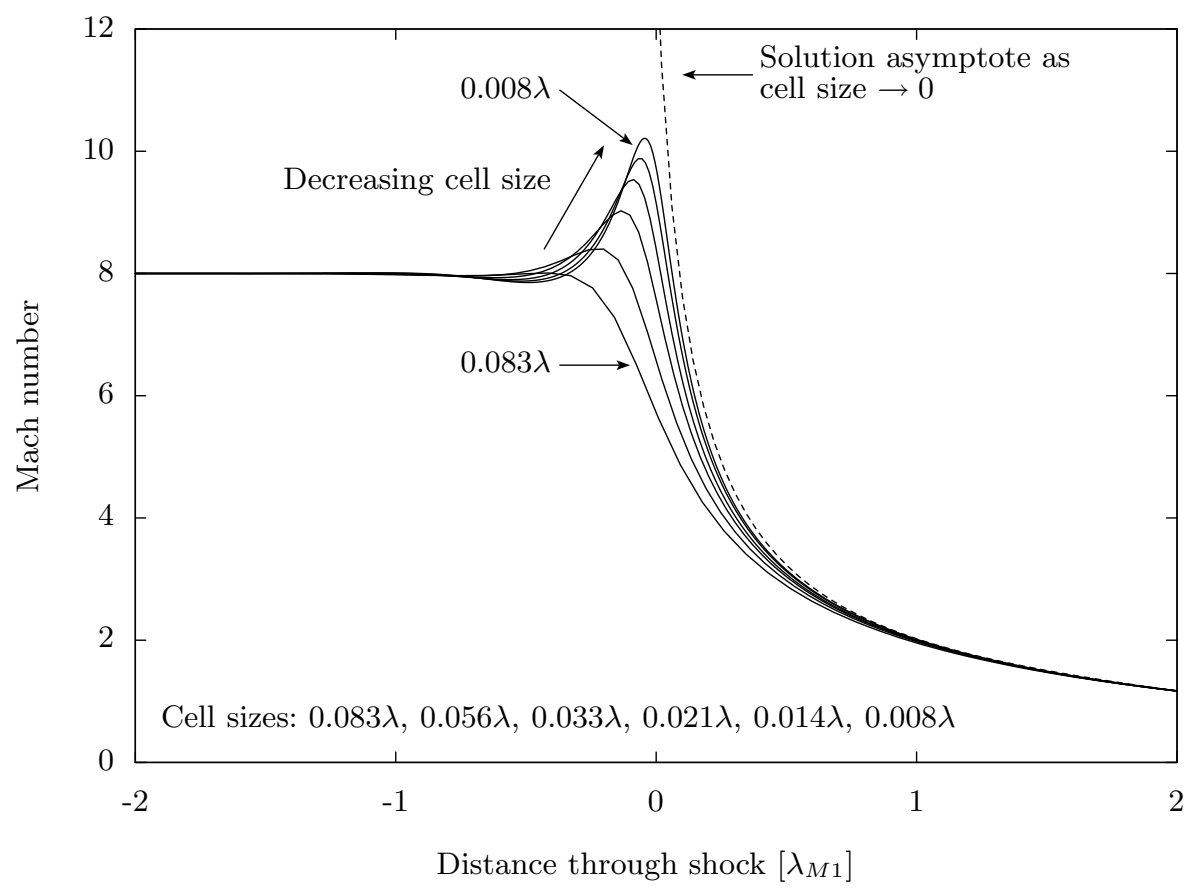

FiguRE 3. Mach number profiles for decreasing cell sizes, assuming $\alpha_{v} \equiv \alpha$.

we reduce the volume diffusivity coefficient to $\alpha_{v}=\nu$ (i.e. when we set $\alpha^{\star}=1$ ) does the overshoot disappear.

\subsection{Investigation of the unphysical behaviour}

Zhong et al. (1991) showed that some forms of extended hydrodynamic equations are unstable in time to periodic spatial disturbances with wavelengths shorter than a critical length that is typically of the order of one mean free path. Such instabilities appear in numerical simulations when the mesh is sufficiently fine to resolve wavelengths shorter than the critical length, i.e. when the numerical cell length is below this critical length. The appearance of an overshoot below a critical level of mesh refinement in our simulations may indicate a similar instability, although the overshoot does appear at a particularly short cell length and the solutions do converge to a steady state and so do not 'blow up' in time.

Similarly, some forms of extended hydrodynamic equations, which may be stable in time, are actually unstable in space to periodic temporal disturbances (see Struchtrup \& Torrilhon 2003; Torrilhon \& Struchtrup 2004; Struchtrup 2005). Again, it is unclear that such an instability would produce the overshoot behaviour we witnessed in our preliminary calculations. Nevertheless, here we undertake both temporal and spatial stability analyses of the Brenner-Navier-Stokes equations in order to investigate the source of unphysical behaviour.

Following the procedures described in Zhong et al. (1991) and Struchtrup \& Torrilhon (2003), the governing equations from section 4 are first linearised in 1-dimension to 
produce the following non-dimensionalised perturbation equations:

$$
\frac{\partial \phi}{\partial t^{\prime}}+\left[\begin{array}{ccc}
0 & 1 & 0 \\
1 & 0 & 1 \\
0 & \frac{2}{3} & 0
\end{array}\right] \frac{\partial \phi}{\partial x^{\prime}}+\frac{\partial}{\partial x^{\prime}}\left\{\begin{array}{c}
0 \\
\sigma^{\prime} \\
\frac{2}{3} q^{\prime}
\end{array}\right\}=0
$$

where $t^{\prime}$ and $x^{\prime}$ are nondimensionalised time and distance, respectively, $\phi=\left\{\rho^{\prime} u^{\prime} T^{\prime}\right\}^{\mathrm{T}}$ is the vector of nondimensionalised density, flow speed and temperature, and $\sigma^{\prime}$ and $q^{\prime}$ are nondimensionalised momentum and heat fluxes respectively. From (4.7) and (4.9), these momentum and energy fluxes are, respectively,

$$
\sigma^{\prime}=-\frac{4}{3} \frac{\partial u^{\prime}}{\partial x^{\prime}}-\frac{4}{3} \alpha^{\star} \frac{\partial^{2} \rho^{\prime}}{\partial x^{\prime 2}}
$$

and

$$
q^{\prime}=-\frac{15}{4} \frac{\partial T^{\prime}}{\partial x^{\prime}}-\alpha^{\star} \frac{\partial \rho^{\prime}}{\partial x^{\prime}}
$$

We assume a solution to (6.1) of the form

$$
\phi=\tilde{\phi} \exp \left\{\mathrm{i}\left(\omega t^{\prime}-k x^{\prime}\right)\right\},
$$

where $\tilde{\phi}$ is the amplitude of the wave, $\omega$ is its frequency and $k$ its propagation constant. Equations (6.1) to (6.4) can be combined to produce a set of linear algebraic equations of the form

$$
\mathcal{A}(\omega, k) \tilde{\phi}=0
$$

for which non-trivial solutions require

$$
\operatorname{det}[\mathcal{A}(\omega, k)]=0 .
$$

For the Brenner-Navier-Stokes equations, (6.6) yields the following characteristic equation:

$$
6 \mathrm{i} \omega^{3}+23 k^{2} \omega^{2}-\left[10 k^{2}+\left(20+8 \alpha^{\star}\right) k^{4}\right] \mathrm{i} \omega-\left[\left(15-4 \alpha^{\star}\right) k^{4}+20 \alpha^{\star} k^{6}\right]=0 .
$$

If a disturbance in space is considered as an initial-value problem, $k$ is real and $\omega=$ $\omega_{r}+\mathrm{i} \omega_{i}$ is complex. The form of (6.4) indicates that stability then requires $\omega_{i} \geq 0$. If a disturbance in time is considered as a problem of signalling from the boundary, $\omega$ is real and $k=k_{r}+\mathrm{i} k_{i}$ is complex. For a wave travelling in the positive $x$ direction, $k_{r}>0$, and stability then requires that $k_{i}<0$. For a wave travelling in the negative $x$ direction, the converse is true: $k_{r}<0$ and stability requires $k_{i}>0$.

We examine temporal stability by solving (6.7) numerically for $\omega$ for values of $k$ in the range $0 \leq k<\infty$. Trajectories of $\omega$ are plotted in the complex plane in figure 4 . Two sets of trajectories are plotted: those for $\alpha_{v} \equiv \alpha$ (corresponding to $\alpha^{\star}=\operatorname{Pr}^{-1}=1.5$ ) and those for $\alpha_{v} \equiv \nu$ (for which $\alpha^{\star}=1.0$ ). In both cases the trajectories all lie within the region $\omega_{i} \geq 0$, indicating stability for all $k$. This confirms, as expected, that the observed overshoot is not caused by temporal instability.

We then turn to examine spatial stability by solving (6.7) numerically for $k$ for values of $\omega$ in the range $0 \leq \omega<\infty$. Trajectories of $k$ are plotted in the complex plane in figure 5 for both $\alpha_{v} \equiv \nu$ and $\alpha_{v} \equiv \alpha$. When $\alpha_{v} \equiv \nu$ the trajectories do not violate the stability condition. However, when $\alpha_{v} \equiv \alpha$, the inset graph shows one trajectory starts from $\omega=0, k_{r}=0$ at $k_{i} \approx 0.55$, enters the unstable region $\left\{k_{r}>0, k_{i}>0\right\}$, and exits into the stable region $\left\{k_{r}<0, k_{i}>0\right\}$ by crossing the $k_{r}=0$ axis at $k_{i} \approx 0.56$. Thus, the stability condition is clearly violated for small $\omega$.

Subsequently we examined trajectories for a number of different $\alpha^{\star}$ and we found that 


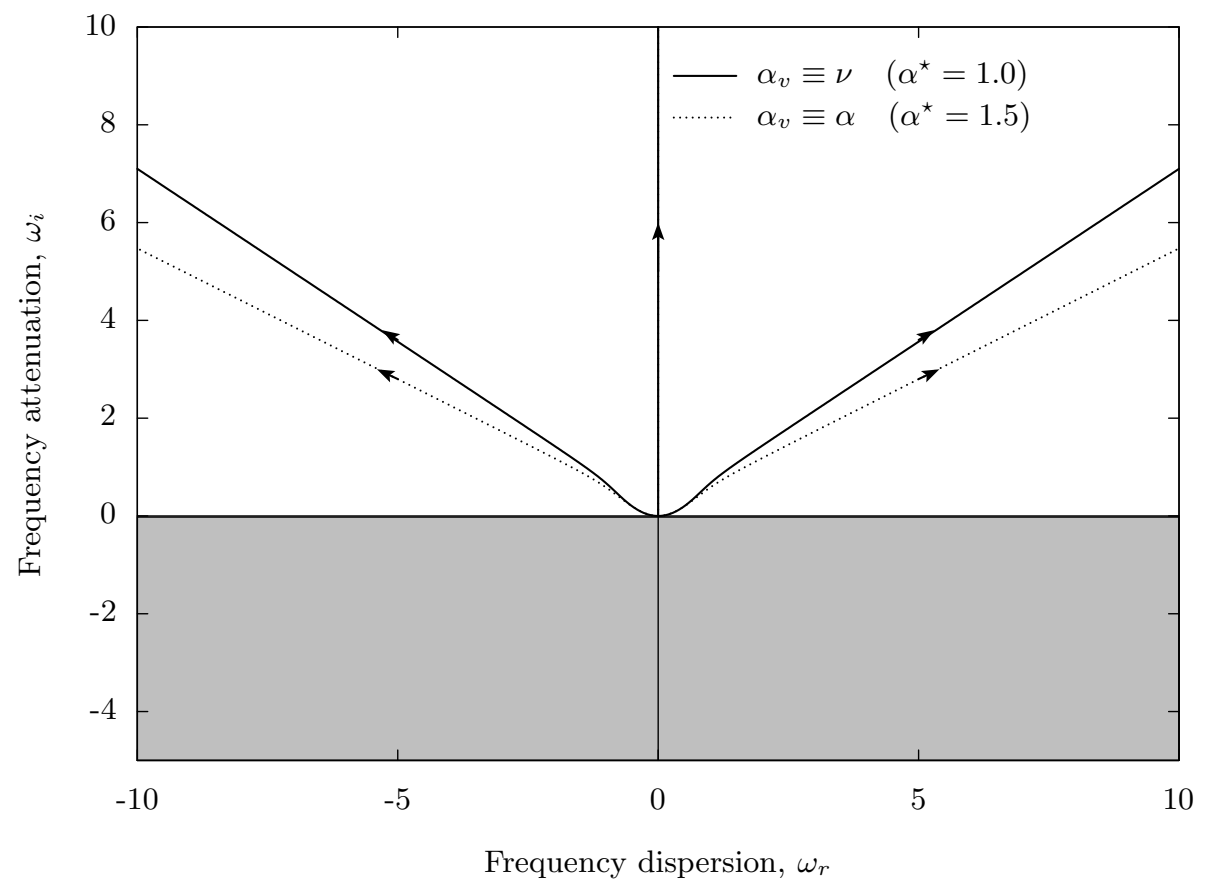

Figure 4. Temporal stability analysis of Brenner-Navier-Stokes equations (arrows indicate direction of increasing $k$; grey shaded area indicates region of instability).

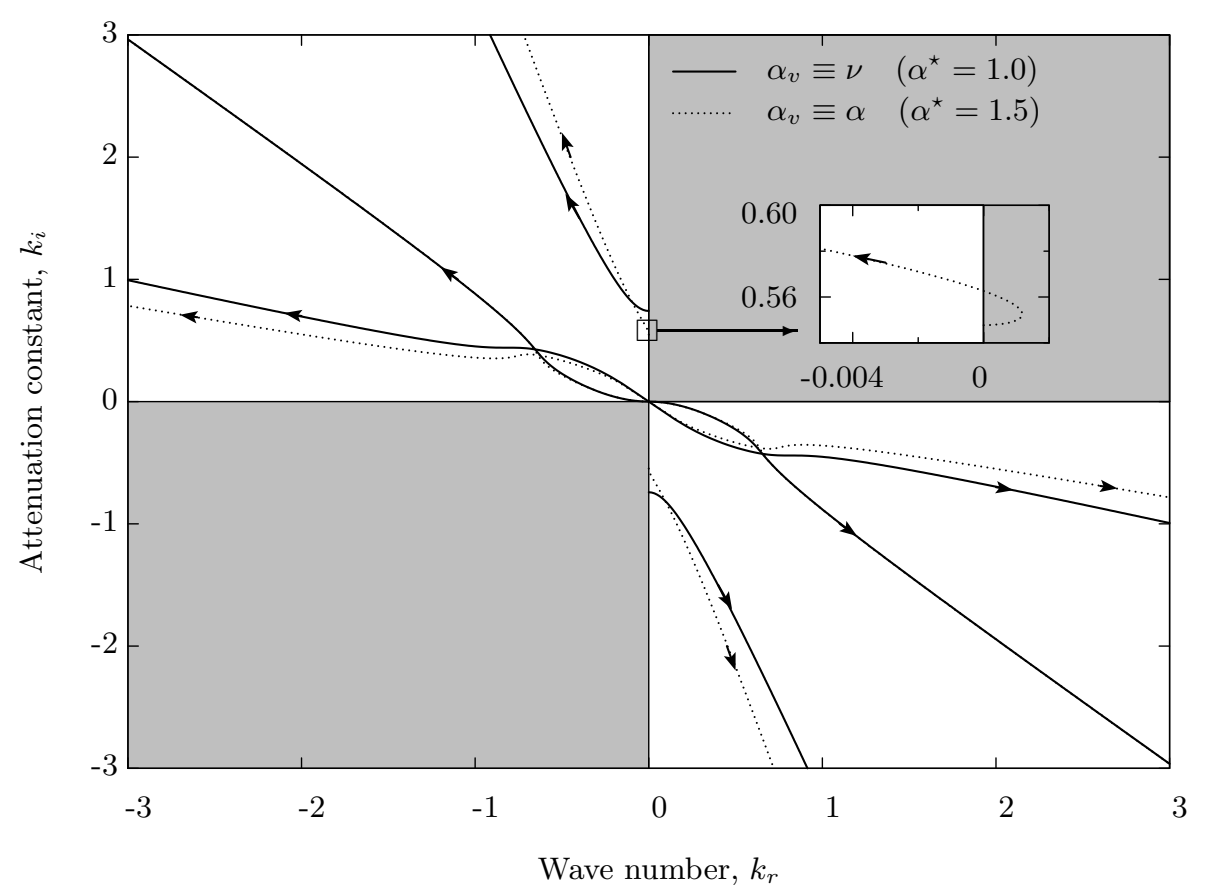

Figure 5. Spatial stability analysis of Brenner-Navier-Stokes equations (arrows indicate direction of increasing $\omega$; grey shaded areas indicate regions of instability). 


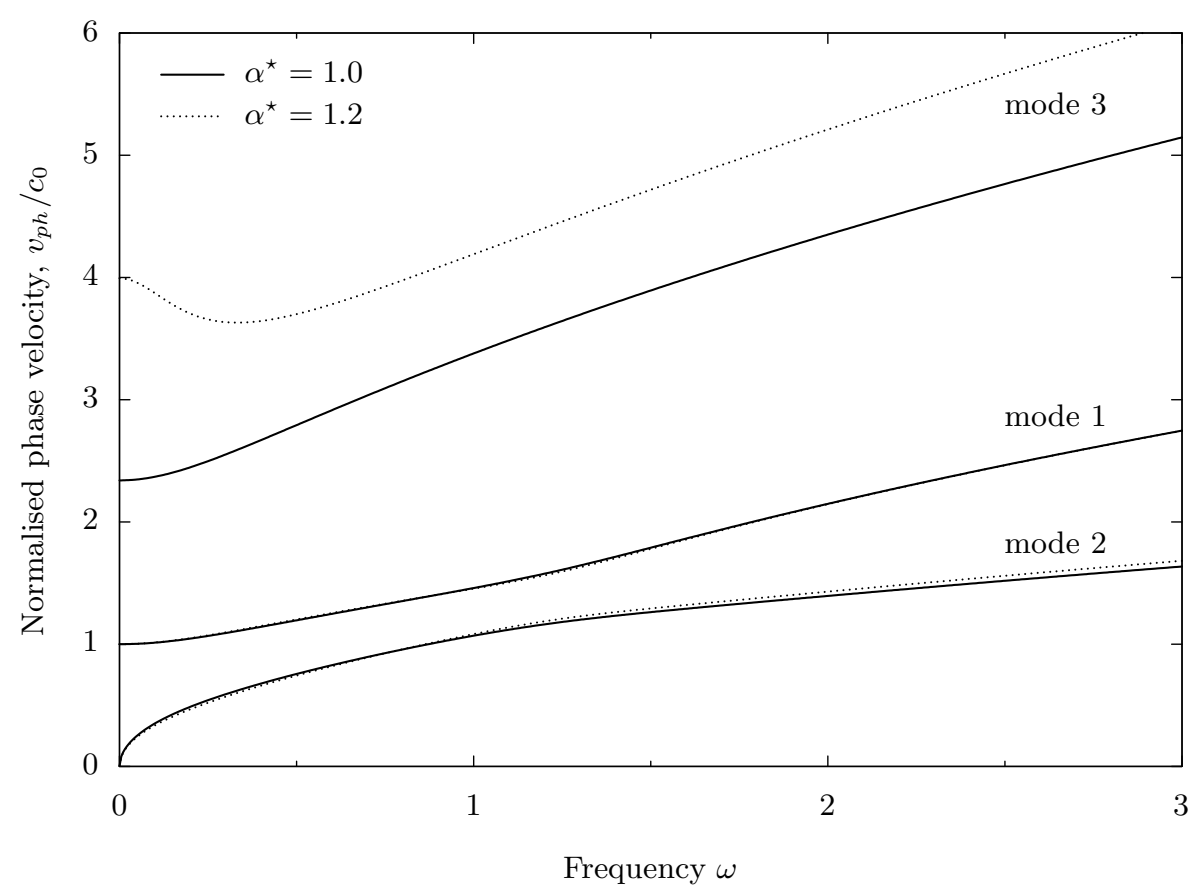

FiguRE 6. Normalised phase velocity for the Brenner-Navier-Stokes equations.

the equations are unstable for $\alpha^{\star} \gtrsim 1.45$, which suggests a potential problem for some intuitive choices of $\alpha_{v}$, such as $\alpha$ and $D_{m}$. However, this result does not really explain the cause of the overshoot in Mach number, since the overshoot only disappears when $\alpha^{\star}$ falls to unity, i.e. considerably lower than 1.45 .

Unphysical behaviour can also be observed by examining the phase velocity:

$$
v_{p h}=\frac{\omega}{k_{r}(\omega)} .
$$

Figure 6 shows the phase velocity, normalised by the speed of sound in the nondimensionalised form in the perturbation equations $\left(c_{0}=\sqrt{\gamma}\right)$, for $\alpha^{\star}=1.0$ and $\alpha^{\star}=1.2$. For both $\alpha^{\star}$, results for mode 1 are superimposed and correspond to the propagation of sound. The mode 2 results correspond to the diffusive transport of heat and results for both $\alpha^{\star}$ are very similar. However, there is a marked difference between results for the two cases for mode 3, relating to higher-order diffusive transport. The $\alpha^{\star}=1.0$ results are similar to those of other extended hydrodynamic models, e.g. the super-Burnett equations (see Struchtrup \& Torrilhon 2003), beginning at a moderate speed, $v_{p h} / c_{0}=2.34$ at $\omega=0$, before increasing steadily with increasing $\omega$. The $\alpha^{\star}=1.2$ results are, however, unusual: the phase velocity at $\omega=0$, i.e. $v_{p h} / c_{0}=3.99$, is high in comparison to other hydrodynamic models (see Struchtrup \& Torrilhon 2003). The phase velocity also decreases initially with increasing $\omega$, before passing through a minimum and increasing thereafter. The high initial phase velocity seems anomalous, and rises to extraordinary levels for higher $\alpha^{\star}$, e.g. if $\alpha^{\star}=1.5$ then $v_{p h} / c_{0}=190.1$ at $\omega=0$. The initial decrease in phase velocity with increasing $\omega$ may allow low frequency waves upstream of the shock to overtake slower, higher frequency waves within the shock, creating counter-dispersion at the upstream end of the shock. The initial decrease in phase velocity disappears only 
when $\alpha^{\star}$ falls below $\sim 1.11$, a level quite close to that at which we find the unphysical overshoot disappears.

To summarise: our results show unphysical behaviour for the Brenner-Navier-Stokes equations when $\alpha^{\star}=1.5$. A spatial stability test confirms the equations are unstable to temporal disturbances when $\alpha^{\star} \gtrsim 1.45$. Plots of phase velocity raise further questions about the physical nature of the solutions when $\alpha^{\star} \gtrsim 1.11$. Taken together, this casts doubt both on Brenner's proposed $\alpha_{v} \equiv \alpha$ and on the apparently natural choice of $\alpha_{v} \equiv D_{m}$. The overshoot in Mach number disappears when $\alpha^{\star}=1.0$, i.e. $\alpha_{v} \equiv \nu$. We therefore adopt this model for $\alpha_{v}$ in the Brenner-Navier-Stokes equations for the remainder of this paper.

\section{Results and comparison with experiment}

\subsection{Shock profiles}

We prefer, where possible, to compare results with actual experiments rather than DSMC simulations, since the latter requires certain assumptions relating to the form of the intermolecular force law. We therefore first make comparison for the variation of density through the shock layer using the actual measured data of Steinhilper (1972), Torecki \& Walenta (1993) and Alsmeyer (1976).

Figure 7 shows the variation of normalised density and temperature, $\rho^{\star}$ and $T^{\star}$ respectively, through an argon gas shock of Mach 2.84 calculated using the Navier-Stokes and Brenner-Navier-Stokes equations with $s=0.72$. The experimental density profile of Torecki \& Walenta (1993) is also shown. It is clear that the shock layer predicted by the conventional Navier-Stokes equations is too thin, whereas the Brenner-Navier-Stokes equations produce good agreement with the experimental data. The main region of disparity is upstream of the shock layer (left hand side in the figure) where the experimental data trails out and is flatter than the prediction.

Similarly, figure 8 shows the predicted profiles for a Mach 8.0 shock compared with experimental density data of Steinhilper (1972). Again, the Navier-Stokes equations produce a shock profile which is too thin when compared with experiment. However, the Brenner-Navier-Stokes equations produce excellent agreement in the central and downstream shock regions $\left(\rho^{\star}>0.2\right)$, only in the upstream region is the predicted profile sharper than the experimental data shows - just as in the Mach 2.84 case.

Figure 9 shows results for a Mach 9.0 shock of Alsmeyer (1976). In this case our observations are very similar to those we make about the Mach 8.0 shock profile, above; this figure is included here for completeness.

\subsection{Inverse density thickness}

Apart from direct comparison of calculated and experimental shock profiles, there are other shock parameters for which experimental and/or independent numerical data is available. The principal parameter is the non-dimensional shock inverse density thickness, defined as:

$$
L_{\rho}^{-1}=\frac{\lambda_{M 1}}{\rho_{2}-\rho_{1}}|\nabla \rho|_{\max } .
$$

In the absence of an a priori characteristic length scale in an unconfined flow, the definition of $K n$ requires a characteristic dimension of a flow structure, in this case the 


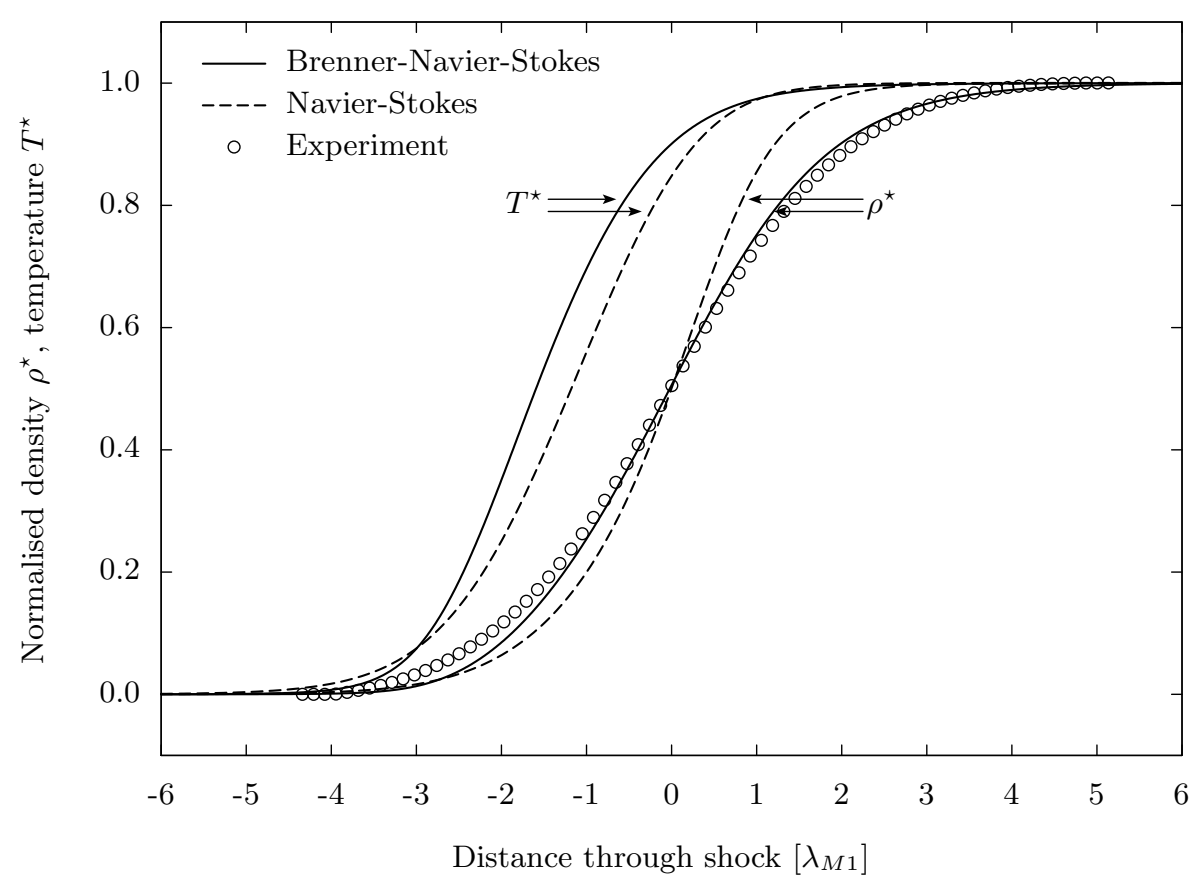

FIGURE 7. Simulated and experimental profiles of a Mach 2.84 stationary shock; $s=0.72$.

actual thickness of the shock layer itself. Therefore $L_{\rho}^{-1}$ has the interesting feature that it represents $K n$ for the shock structure case $\dagger$.

Alsmeyer (1976) reported the most comprehensive collection of experimental shock data, comprising previously-published work as well as his own new results. Figure 10 shows $L_{\rho}^{-1}$ for argon shocks up to Mach 11, with experimental data collated from Steinhilper (1972), Alsmeyer (1976) and Torecki \& Walenta (1993). The Navier-Stokes equations, with $s=0.72$, predict shocks of approximately half the measured thicknesses over the entire Mach number range. As $L_{\rho}^{-1}$ indicates $K n$, this poor agreement is expected because we can see that $K n \sim 0.2-0.25$ over most of this Mach number range, so the Navier-Stokes equations are beyond their effective range of application. However, the results from the Brenner-Navier-Stokes equations closely match experiment, with moderate sensitivity to the choice of viscosity-temperature exponent: using 0.72 (the control value) and 0.76 (our best-fit value for temperatures equivalent to shocks up to Mach 12.5), the results fall within the limits of experimental scatter; using $s=0.68$, the results stray slightly outside the scatter of experimental data just before they reach the exponent's limit of applicability at Mach 4.3. With the results for 0.72 at the higher end of the experimental scatter, and results for 0.76 at the lower end, we estimate that an exponent of $s \approx 0.74$ would produce the best agreement with the experimental results.

\subsection{Density asymmetry quotient}

Agreement of predicted and experimental shock inverse density thicknesses is not the only measure of the success of a new model. As $L_{\rho}^{-1}$ depends on the density gradient at

$\dagger$ While this identification then indicates, as we see below, that shocks generally have such a high overall $K n$ that any hydrodynamic model should fail, we can still assess extended hydrodynamic equations for their usefulness as engineering models. 


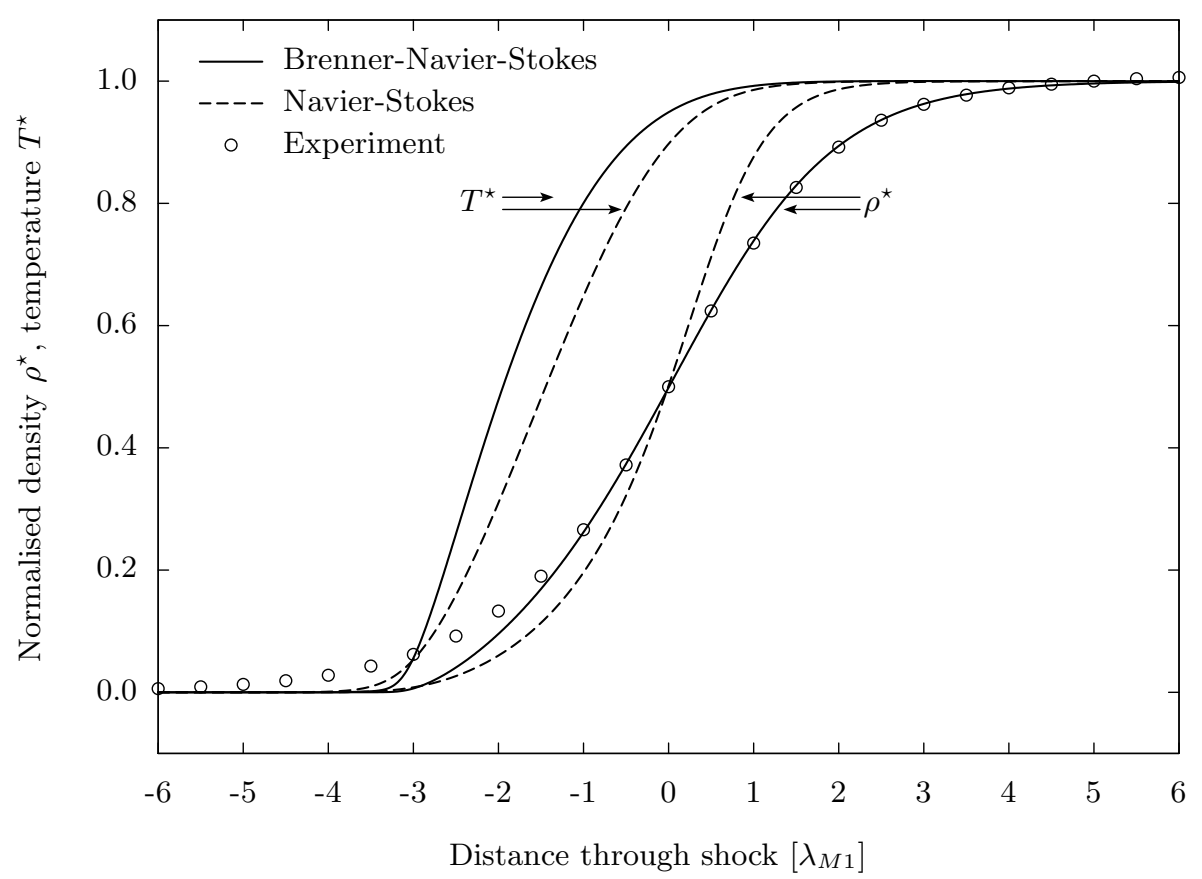

Figure 8. Simulated and experimental profiles of a Mach 8.0 stationary shock; $s=0.72$.

the profile midpoint alone, it does not express anything about the overall shape of the profile. If $L_{\rho}^{-1}$ is used as the sole parameter to describe the shock it could be concluded that the Brenner-Navier-Stokes equations tested here, and many other models previously published, have excellent predictive capability. However, the shock profiles in figures 7 and 8 show there are differences between simulation and experiment, in particular relating to the flatter region upstream of the profile that is observed experimentally.

Therefore, a second parameter which should be used to describe the shock profile, and for which experimental data is available, is the density asymmetry quotient $Q_{\rho}$. This is a measure of how skewed the shock density profile is relative to its midpoint. It is defined for a 1-dimensional profile of normalised density, $\rho^{\star}$, centred at $\rho^{\star}=0.5$ on $x=0$, as

$$
Q_{\rho}=\frac{\int_{-\infty}^{0} \rho^{\star}(x) \mathrm{d} x}{\int_{0}^{\infty}\left[1-\rho^{\star}(x)\right] \mathrm{d} x} .
$$

A symmetric shock would consequently have $Q_{\rho}=1$, but real shock waves are not completely symmetrical about their midpoint. First, their 'bulk' form is generally skewed somewhat towards the downstream. Then, the aforementioned flattened, diffusive region, that extends upstream of the shock profile, tends to increase $Q_{\rho}$. Figure 11 shows experimental data compiled by Alsmeyer (1976) in which $Q_{\rho}$ increases fairly linearly from $\sim 0.9$ at around Mach 1.5, through unity at around Mach 2.3, to $\sim 1.15$ at Mach 9 . The bulk form therefore corresponds to $Q_{\rho} \approx 0.9$ and the upstream flattened region accounts for a further increase in $Q_{\rho}$, of up to 0.25 at Mach 9 .

Results from the Navier-Stokes equations do not agree well with experimental data: the bulk form is skewed towards the upstream side so that $Q_{\rho}>1$ even at the lowest Mach numbers and the skewness further increases with Mach number (apparently by 


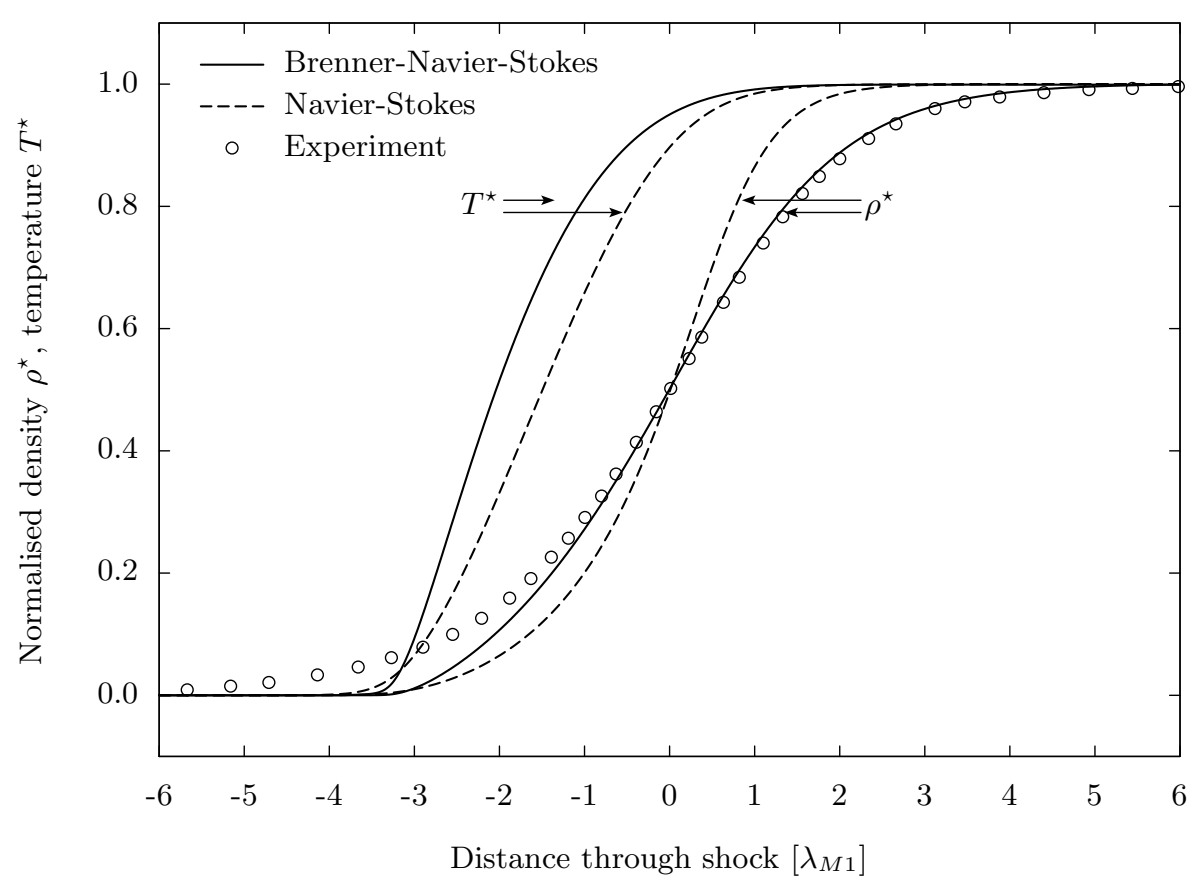

FiguRE 9. Simulated and experimental profiles of a Mach 9.0 stationary shock; $s=0.72$.

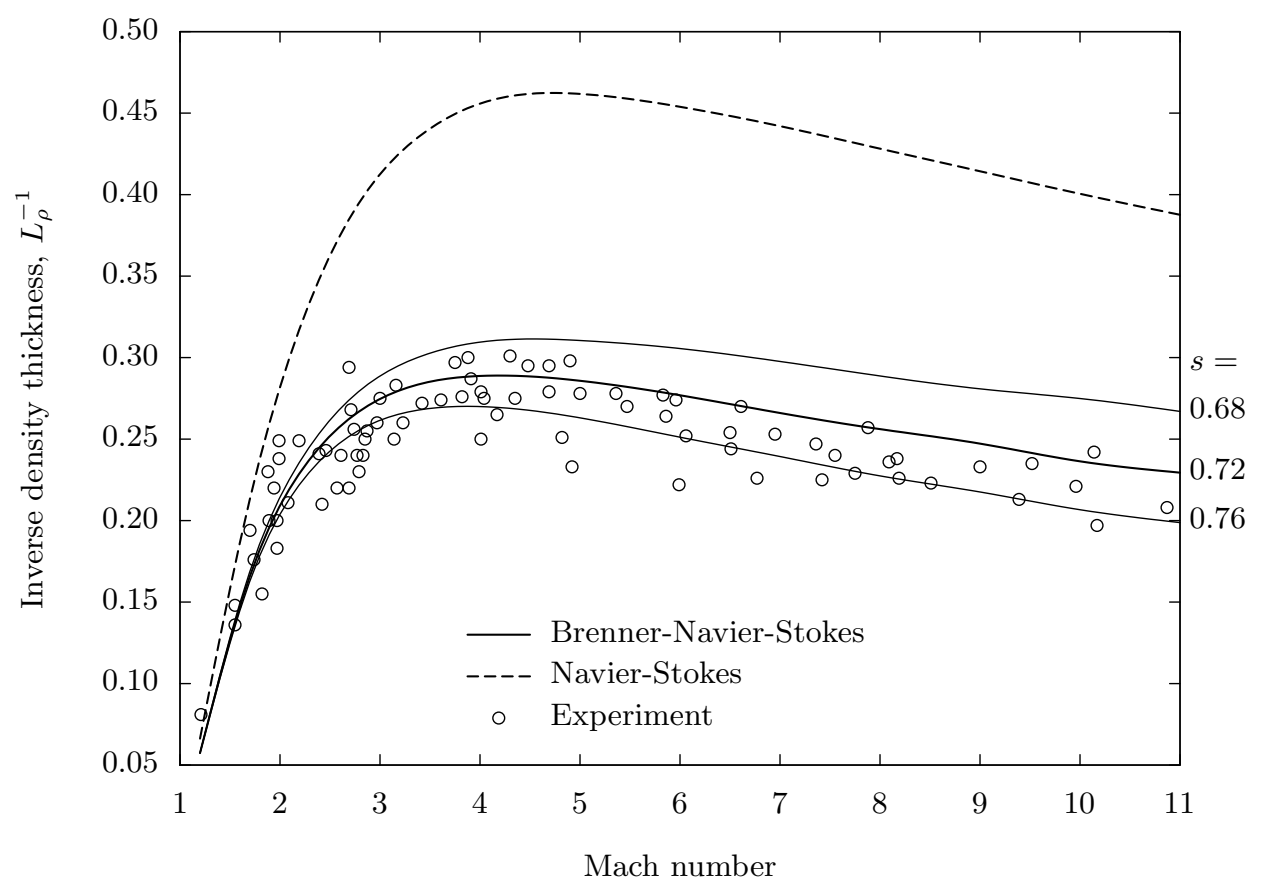

Figure 10. Simulated and experimental inverse density thickness $\left(L_{\rho}^{-1}\right)$ data, versus shock Mach number; for various values of the exponent $s$. 


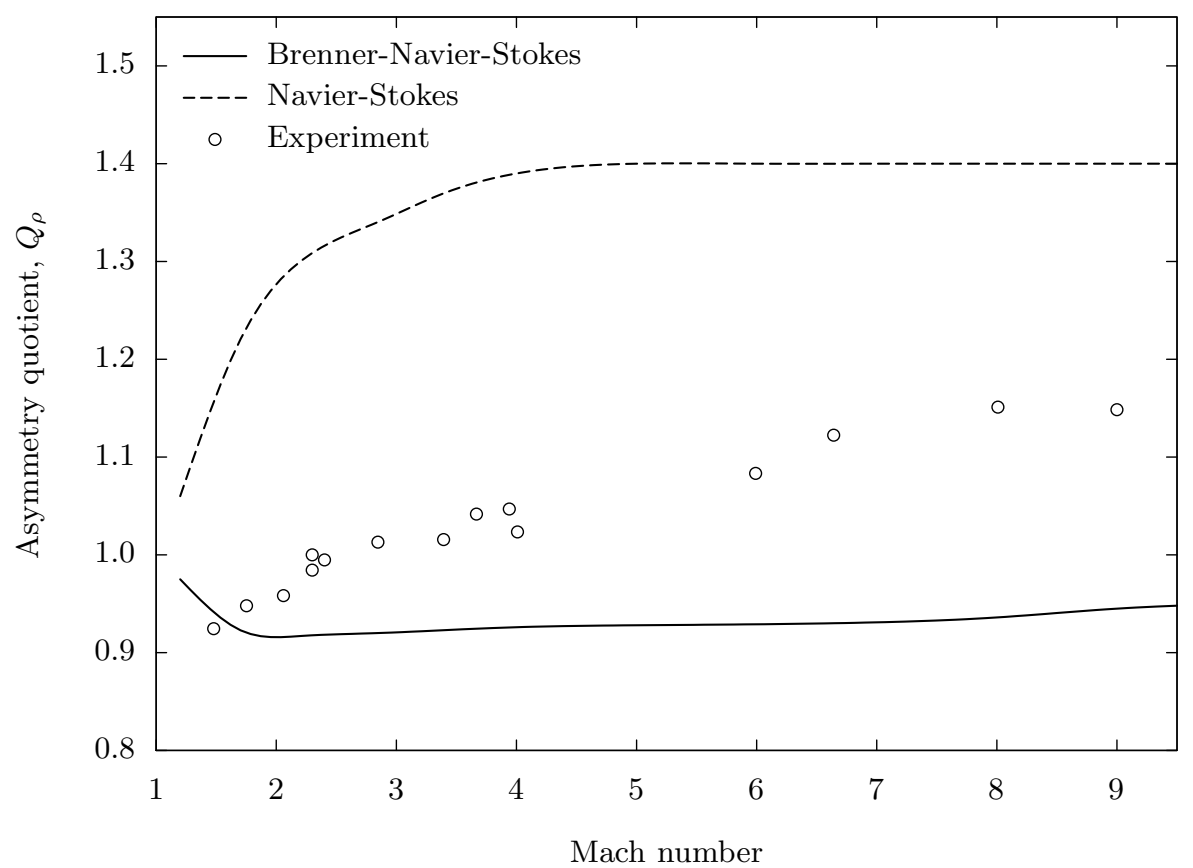

FigURE 11. Simulated and experimental asymmetry quotient $\left(Q_{\rho}\right)$ data, versus shock Mach number; $s=0.72$.

sharpening of the profile downstream rather than flattening upstream) so that by Mach $4, Q_{\rho} \approx 1.4$, compared to $\sim 1.03$ from experiment.

We find the Brenner-Navier-Stokes equations predict the bulk form of the profile very well, predicting $Q_{\rho} \approx 0.9$ at low Mach numbers. As discussed in section 7.1, it does not capture the flattened region upstream and so the departure from experimental data increases with Mach number.

\subsection{Temperature-density separation}

The final shock structure parameter is the temperature-density separation, $\delta_{T \rho}$, measured between the midpoints of the respective normalised profiles. In a shock, the density rises from its upstream value to its downstream value behind the temperature, due to the finite relaxation times for momentum and energy transport; a good hydrodynamic model should resolve this spatial lag accurately. However, experimental data for this parameter is scarce due to the difficulty in measuring temperature profiles, so independent DSMC data is usually taken for comparison.

Figure 12 shows the temperature and density profiles for a Mach 11 shock calculated using DSMC by Lumpkin \& Chapman (1991), and the Navier-Stokes and the BrennerNavier-Stokes models. As in our earlier comparisons in section 7.1, the Brenner-NavierStokes equations produce profiles that are much sharper in the upstream region of the shock than those from DSMC results.

Figure 13 compares DSMC data for $\delta_{T \rho}$ over a range of shock Mach numbers with results from our simulations. The DSMC data show an increase in $\delta_{T \rho}$ from $\sim 1.5 \lambda_{M 1}$ at Mach 1.5 to $\sim 2.9 \lambda_{M 1}$ at Mach 8. The Navier-Stokes and Brenner-Navier-Stokes equations increasingly under-predict $\delta_{T \rho}$ with increasing Mach number, although the 


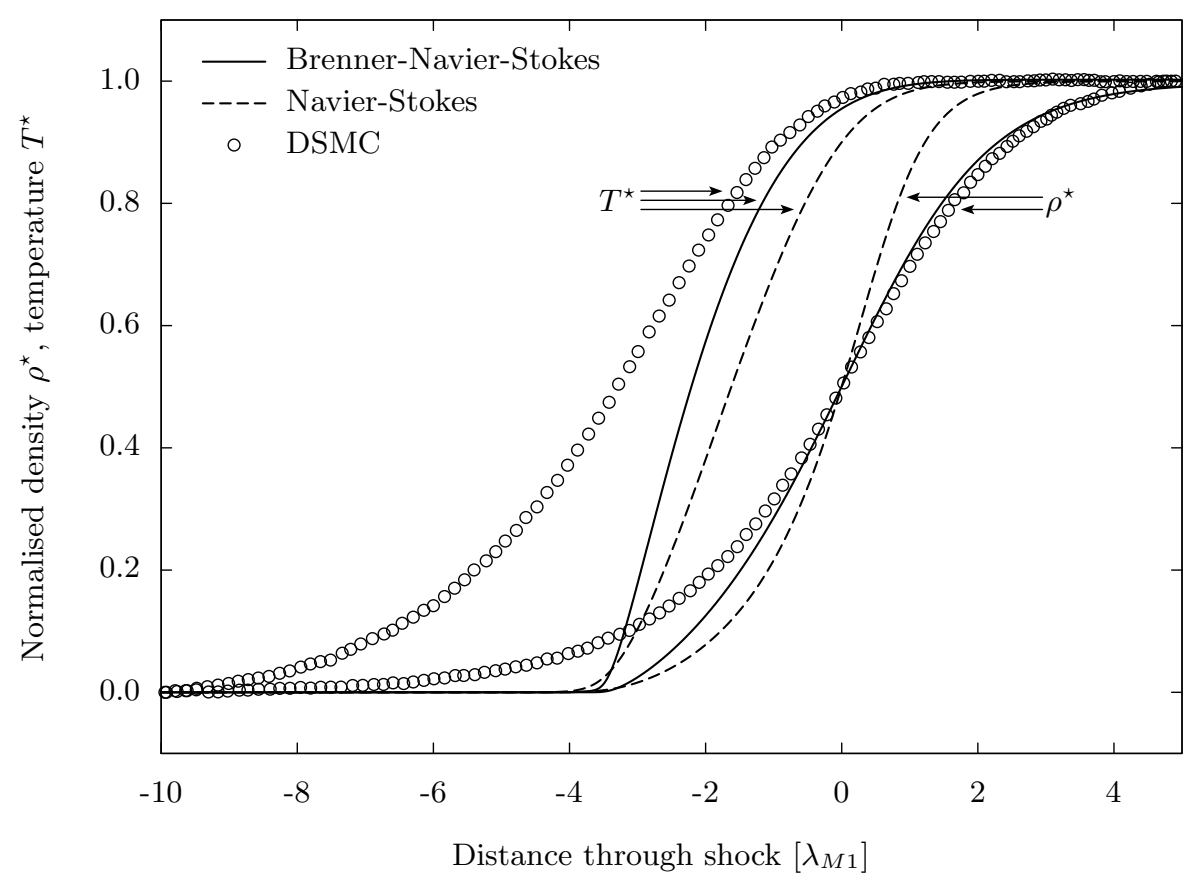

FIGURE 12. Simulated and DSMC profiles of a Mach 11 stationary shock; $s=0.72$.

Brenner-Navier-Stokes equations generally perform a little better over the Mach number range.

\subsection{Very strong shocks}

The inverse density thickness of extremely strong shocks is a useful additional comparison for any proposed hydrodynamic model. Narasimha \& Das (1986) examined the solution of the Boltzmann equation for an infinitely strong shock (a more recent treatment is in Cercignani et al. 1999), modelling the upstream flow as a molecular beam with a distribution function of the form $f(x=-\infty)=n_{1} \delta\left(\boldsymbol{u}_{1}\right)$, where $\delta$ is the Kronecker delta function. The shock layer may then be treated as a device for converting this beam function into a downstream Maxwellian distribution function. The distribution function in the shock layer can be expressed as a linear combination of the two extremal distribution functions, a method similar to the bimodal method of Muckenfuss (1962).

Using an expansion parameter that measures the departure of the distribution function in the shock wave from that outlined in the previous paragraph, an infinite series of ordinary differential equations is obtained for the shock thickness. This series rapidly converges, and a solution of the first seven equations of the set yields a predicted shock thickness of $6.7 \lambda_{M 2}$ (which is written in terms of the downstream Maxwellian mean free path version of 2.1). When this is converted into the $L_{\rho}^{-1}$ of (7.1), the inverse density thickness for a shock with a downstream $\lambda_{M 2}$ equivalent to that of a very strong shock of Mach 100 is predicted to be 0.076 .

Our calculations for shocks of Mach 100 give $L_{\rho}^{-1}=0.156$ for the Navier-Stokes equations with $s=0.72$. The results with the Brenner-Navier-Stokes equations are $L_{\rho}^{-1}=0.091$ for $s=0.72$, and $L_{\rho}^{-1}=0.066$ for $s=0.76$. These values for $L_{\rho}^{-1}$ straddle the solution from the molecular beam analysis. Further simulations with successive adjustments to $s$ gave a precise match in $L_{\rho}^{-1}$ for $s=0.742$, which is in agreement with the 


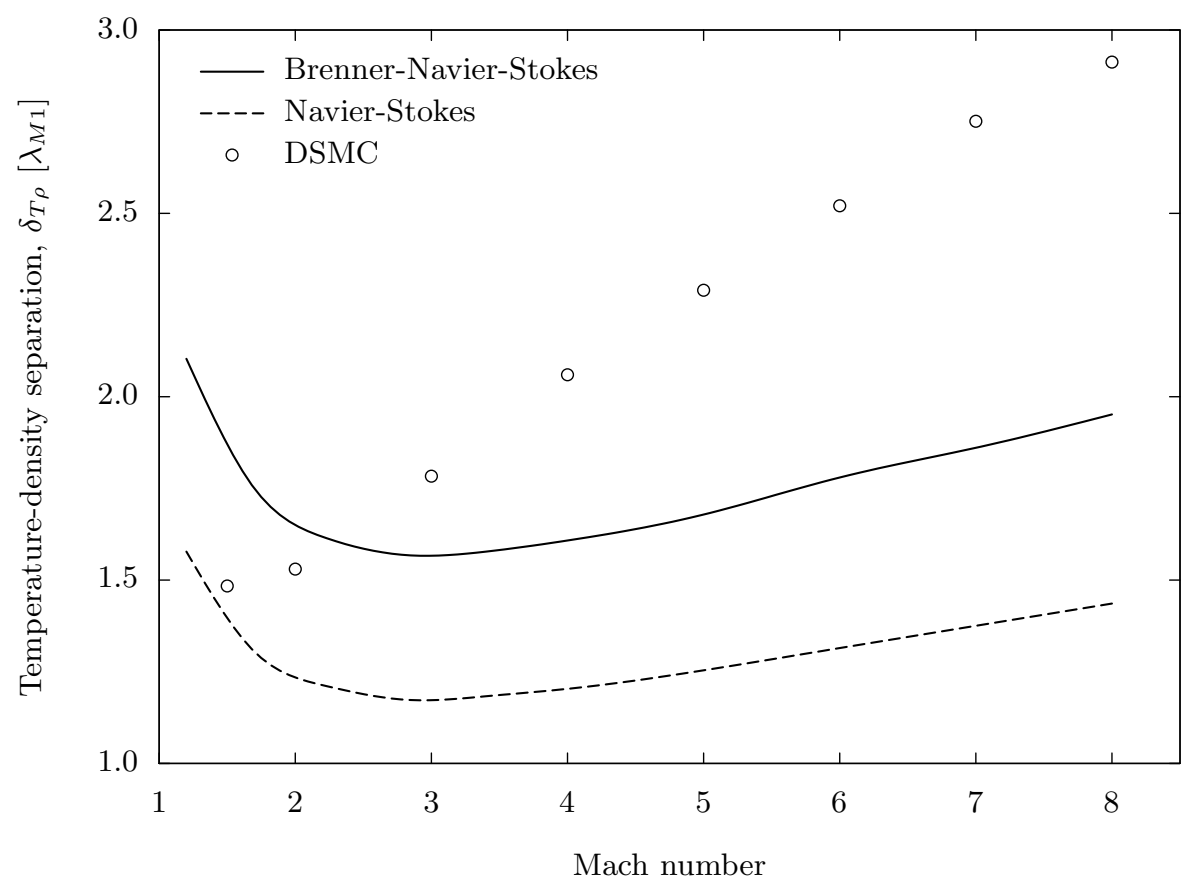

Figure 13. Simulated and independent DSMC temperature-density separation $\left(\delta_{T \rho}\right)$ data, versus shock Mach number; $s=0.72$.

exponent estimated in section 7.2 to produce the best agreement with experiment over the range Mach 1-11.

\section{Discussion and conclusions}

The Navier-Stokes equations are robust and accurate over a wide range of $\mathrm{Kn}-$ surprisingly so, given some of the relatively narrow axioms on which they depend (i.e. the continuum-fluid and local-equilibrium requirements). Such a good fluids engineering model is difficult to relinquish, even when flow systems well beyond its range of applicability are considered (see, e.g., Herwig \& Hausner 2003). However, it is clear from the results in section 7 that the Navier-Stokes equations fail in nearly every respect in predicting correct shock structures above about Mach 2 (or, equivalently, for intermediate- $K n$ flows).

While it is important not to draw strong conclusions based on just one test case, our results are generally encouraging for the Brenner-Navier-Stokes equations. This modified model is significantly better at reproducing the trends in the experimental and DSMC data, and in the case of the inverse density thickness delivers an excellent match. It is only the more detailed features of the shock profile that Brenner's model seems unable to reproduce.

First, it does not predict the flattened upstream region, as discussed in section 7.3. In this regard, a major advantage of DSMC as a technique for simulating intermediate$K n$ flows in general is its ability to produce non-Maxwellian velocity distributions, that may also differ in directions parallel and perpendicular to the flow. It is not clear that hydrodynamic models will be able to properly incorporate this physics, and certainly the problems the Brenner-Navier-Stokes equations have in capturing the upstream shock 
region properly is related to the fact that in this region the velocity distribution function is a non-Maxwellian combination of fast, cold upstream molecules and slower, hot molecules that have diffused from downstream regions.

The second feature is also related to this distribution function problem: bi-modal methods (see, e.g., Cercignani et al. 1999) for a hard-sphere gas predict a downstream temperature overshoot of around 1\%, which is confirmed by careful DSMC simulations. There are no downstream overshoots predicted in any of the Brenner-Navier-Stokes shock simulations.

While some of these features can be obtained using certain extended hydrodynamic models that are formally $O\left(K n^{2}\right)$ (see, e.g., Lumpkin \& Chapman 1991; Reese 1993; Reese et al. 1995), this is at a cost: there are known problems of physical stability, and the numerical implementation is difficult due to the large number of additional non-linear and high-order derivatives. The Brenner modification does not suffer as much from these problems, having only a single additional term in each of the momentum and energy conservation equations. That the adoption of these terms provides a substantial improvement in predicted results raises the question of whether this model can compensate, in part at least, for increased non-local-equilibrium in the gas, or whether this agreement is coincidental.

Brenner $(2005 b)$ proposed his modifications partly to understand how some effects that are traditionally thought of as becoming important only in a flow approaching the intermediate- $K n$ range, e.g. slip at solid bounding surfaces, can be encompassed in a model which still retains its essential $O(K n)$ character. He shows that the form (if not the exact coefficients, except for a certain molecular model) of two particular terms that appear in the Burnett constitutive model for $\boldsymbol{T}$ are in fact encompassed by the additional term in $\rho$ in his modified Newtonian $\boldsymbol{T}$ of (4.7). While all the stress terms in the Burnett equations are formally $O\left(K n^{2}\right)$, under some circumstances these two particular terms can be of similar magnitude to those of $O(K n)$ i.e. the same order of accuracy as the Navier-Stokes equations. If the issue of the correct model for the volume diffusivity, $\alpha_{v}$, can be resolved then the Brenner-Navier-Stokes equations may therefore provide a simple alternative to the family of extended hydrodynamic models that includes those of Burnett, Grad etc., producing reasonably accurate solutions of intermediate- $K n$ flows at a modest computational cost.

While it is known that the classical Burnett equations do not satisfy the second law of thermodynamics, truncated or extended forms of the equations can be constructed that do (see, e.g., Lumpkin \& Chapman 1991; Zhong et al. 1991; Reese 1993; Reese et al. 1995). The fact that the Brenner-Navier-Stokes equations are less prone to both numerical instability and unphysical solution may indicate that thermodynamic consistency is less of a problem with these equations than with more complex extended hydrodynamics models.

We recognise that it is not reasonable to rely on one benchmark case to decide the value of the Brenner-Navier-Stokes equations, or any other extended hydrodynamic model (or its associated boundary conditions). Equation (1.1) indicates there are three distinct categories of near-equilibrium flows:

A. $M a=O(1), R e \rightarrow \infty$, typical of super- and hypersonic flows;

B. $M a \rightarrow 0, R e=O(1)$, typical of flows in micro- or nano-systems;

C. $M a \rightarrow 0, R e \rightarrow \infty$, typical of incompressible turbulent boundary layer flows.

As $K n$ vanishes more quickly for flows in category $\mathrm{C}$ than in categories $\mathrm{A}$ and $\mathrm{B}$, departures from local equilibrium in category $\mathrm{C}$ flows are not as significant as those in category A or B flows. This paper has addressed a category A flow in which the boundary conditions are not in doubt, but benchmark cases for models of intermediate- $K n$ flows 
generally require additional boundary conditions, usually at solid surfaces, the specification of which is one of the outstanding problems in hydrodynamic approaches to rarefied gas dynamics. Setting aside the boundary condition problem, however, we can propose a number of benchmark cases in categories A and B that any new hydrodynamic model for rarefied flows should be tested against:

- the shock structure problem, as outlined in this paper (including comparisons of $Q_{\rho}$, $\delta_{T \rho}$ and the thickness of $M a=\infty$ shocks, in addition to the usual comparison with $\left.L_{\rho}^{-1}\right)$;

- the nonlinearity of the thermal and momentum Knudsen layers (the region $O(\lambda)$ close to solid surfaces);

- the 'Knudsen paradox' in Poiseuille flow, i.e. the minimum in the mass flow rate at around $K n \approx 1$, as well as bimodality in the temperature profile;

- drag coefficients and heat transfer in: flow around a sphere, flow around a cylinder and Couette flow;

- variation of skin friction on cones in supersonic flow;

- base pressures on cone-cylinder configurations in supersonic flow as the Knudsen layer extends far into the wake region;

- thermophoresis, i.e. the force on particles suspended in a rarefied gas between two parallel plates of different temperature;

- dispersion and absorption of ultrasonic sound waves.

This list is neither exclusive nor comprehensive; we are sure that other good benchmark cases could be added to it. The caveat is that in most cases experimental data is extremely sparse and unreliable, and unfortunately much reliance still needs to be placed on comparison with independent DSMC or other molecular dynamics simulations as 'experimental analogues'.

We would like to thank Steve Daley of Dstl Farnborough (UK), Howard Brenner of MIT (USA), Henry Weller of OpenCFD Ltd. (UK) and Duncan Lockerby of Warwick University (UK) for useful discussions. We also thank the referees of this paper for their very helpful comments. This work is funded in the UK by the Engineering and Physical Sciences Research Council under grant GR/T05028/01, and through a Philip Leverhulme Prize for JMR from the Leverhulme Trust.

\section{REFERENCES}

Aeschliman, D. P. \& Cambel, A. B. 1970 Properties of gases at very high temperatures. Physics of Fluids 13, 2466.

Alsmeyer, H. 1976 Density profiles in argon and nitrogen shock waves measured by the absorption of an electron beam. Journal of Fluid Mechanics 74, 497.

Amdur, I. \& Mason, E. A. 1958 Properties of gases at very high temperatures. Physics of Fluids 1, 370

BARCELO, B. T. 1971 Electron beam measurements of the shock wave structure: Part 1, Determination of the interaction potential of the noble gases from shock wave structure experiments. PhD thesis, California Institute of Technology, USA.

Bardow, A. \& Öttinger, H. C. 2007 Consequences of the Brenner modification to the NavierStokes equations for dynamic light scattering. Physica A 373, 88-96.

Bird, G. A. 1970 Aspects of the structure of strong shock waves. Physics of Fluids 13, 1172.

Brenner, H. 2005a Kinematics of volume transport. Physica A 349, 11.

Brenner, H. $2005 b$ Navier-Stokes revisited. Physica A 349, 60.

Cercignani, C., Frezzotti, A. \& Grosfils, P. 1999 The structure of an infinitely strong shock wave. Physics of Fluids 11, 2757.

Chapman, S. \& Cowling, T. G. 1970 The mathematical theory of non-uniform gases, 3rd edn. Cambridge, UK: Cambridge University Press. 
Herwig, H. \& Hausner, O. 2003 Critical view on "new results in micro-fluid mechanics": an example. International Journal of Heat and Mass Transfer 46, 935.

Hirsch, C. 1990 Numerical computation of internal and external flows, , vol. 2. Chichester, UK: John Wiley and Sons Ltd.

IssA, R. I. 1986 Solution of the implicitly discretised fluid flow equations by operator-splitting. Journal of Computational Physics 62, 40-65.

VAN LEeR, B. 1979 Towards the ultimate conservative difference scheme, V: a second-order sequel to Godunov's method. Journal of Computational Physics 32, 101-136.

Levermore, C. D. \& Morokoff, W. J. 1998 The Gaussian moment closure for gas dynamics. SIAM Journal of Applied Mathematics 59, 72.

OpEnCFD LTD 2004 http://www.openfoam.org.

Lumpkin, F. E. \& Chapman, D. R. 1991 Accuracy of the Burnett equations for hypersonic real gas flows. AIAA Paper 91-0771.

Macrossan, M. N. \& Lilley, C. R. 2003 Viscosity of argon at temperatures $>2000$ K from measured shock thickness. Physics of Fluids 15, 3452.

Maitland, G. C. \& Smith, E. B. 1972 Critical reassessment of viscosities of 11 common gases. Journal of Chemical Engineering Data 17, 150.

Muckenfuss, C. 1962 Some aspects of shock structure according to the bimodal model. Physics of Fluids 5, 1325.

Myong, R. S. 2001 A computational method for Eu's generalized hydrodynamic equations of rarefied and microscale gas. Journal of Computational Physics 168, 47.

NARAsimha, R. \& DAs, P. 1986 The infinitely strong shock. In 15th International Symposium on Rarefied Gas Dynamics (ed. V. Boffi \& C. Cercignani), pp. 293-307. B. G. Teubner, Stuttgart, Germany.

Öttinger, H. C. 2005 Beyond equilibrium thermodynamics. Hoboken, USA: John Wiley and Sons.

ReEse, J. M. 1993 On the structure of shock waves in monatomic rarefied gases. PhD thesis, Oxford University, UK.

Reese, J. M., Woods, L. C., Thivet, F. J. P. \& CAndel, S. M. 1995 A second-order description of shock structure. Journal of Computational Physics 117, 240-250.

Rhie, C. M. \& Chow, W. L. 1982 A numerical study of the turbulent flow past an isolated airfoil with trailing edge separation. AIAA-82-0998, AIAA/ASME 3rd Joint Thermophysics, Fluids, Plasma and Heat Transfer Conference, St. Louis, Missouri.

SchaAf, S. A. \& Chambré, P. L. 1961 Flow of rarefied gases. USA: Princeton University Press.

Steinhilper, E. A. 1972 Electron beam measurements of the shock wave structure: Part 1, The inference of intermolecular potentials from shock structure experiments. PhD thesis, California Institute of Technology, USA.

Struchtrup, H. 2005 Macroscopic transport equations for rarefied gas flows. Heidelberg, Germany: Springer.

Struchtrup, H. \& Torrilhon, M. 2003 Regularization of Grad's 13 moment equations: Derivation and linear analysis. Physics of Fluids 15, 2668.

Sturtevant, B. \& Steinhilper, E. A. 1986 Intermolecular potentials from shock structure. In 8th International Symposium on Rarefied Gas Dynamics, p. 159.

Torecki, P. \& Walenta, Z. 1993 Private communication. Polish Academy of Sciences, Warsaw, Poland.

Torrilhon, M. \& Struchtrup, H. 2004 Regularized 13-moment equations: shock structure calculations and comparison to Burnett models. Journal of Fluid Mechanics 513, 171.

Xu, K. \& TANG, L. 2004 Nonequilibrium Bhatnagar-Gross-Krook model for nitrogen shock structure. Journal of Computational Physics 16, 3824.

Zhong, X., MacCormack, R. W. \& Chapman, D. R. 1991 Stabilisation of the Burnett equations and application to high-altitude hypersonic flows. AIAA Paper 91-0770. 